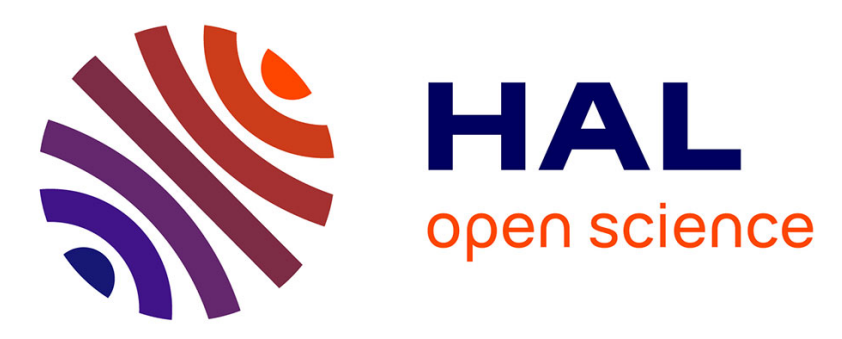

\title{
An objective meta-modeling approach for indentation-based material characterization
}

Liang Meng, Balaji Raghavan, Olivier Bartier, Xavier Hernot, Gerard Mauvoisin, Piotr Breitkopf

\section{- To cite this version:}

Liang Meng, Balaji Raghavan, Olivier Bartier, Xavier Hernot, Gerard Mauvoisin, et al.. An objective meta-modeling approach for indentation-based material characterization. Mechanics of Materials, 2017, 107, pp.31-44. 10.1016/j.mechmat.2017.01.011 . hal-01451417

\section{HAL Id: hal-01451417 \\ https://hal.science/hal-01451417}

Submitted on 4 Jul 2017

HAL is a multi-disciplinary open access archive for the deposit and dissemination of scientific research documents, whether they are published or not. The documents may come from teaching and research institutions in France or abroad, or from public or private research centers.
L'archive ouverte pluridisciplinaire $\mathbf{H A L}$, est destinée au dépôt et à la diffusion de documents scientifiques de niveau recherche, publiés ou non, émanant des établissements d'enseignement et de recherche français ou étrangers, des laboratoires publics ou privés. 


\section{Highlights}

- Indentation imprint mapping was applied to the problem of characterization of plastic behavior of materials.

- Image-comparison approach based on supervised nonlinear manifold learning.

- The protocol was designed to avoid the usual problems associated with the use of the loadingunloading curve.

- The protocol was applied on three different materials and the results obtained were compared with direct tensile testing. 


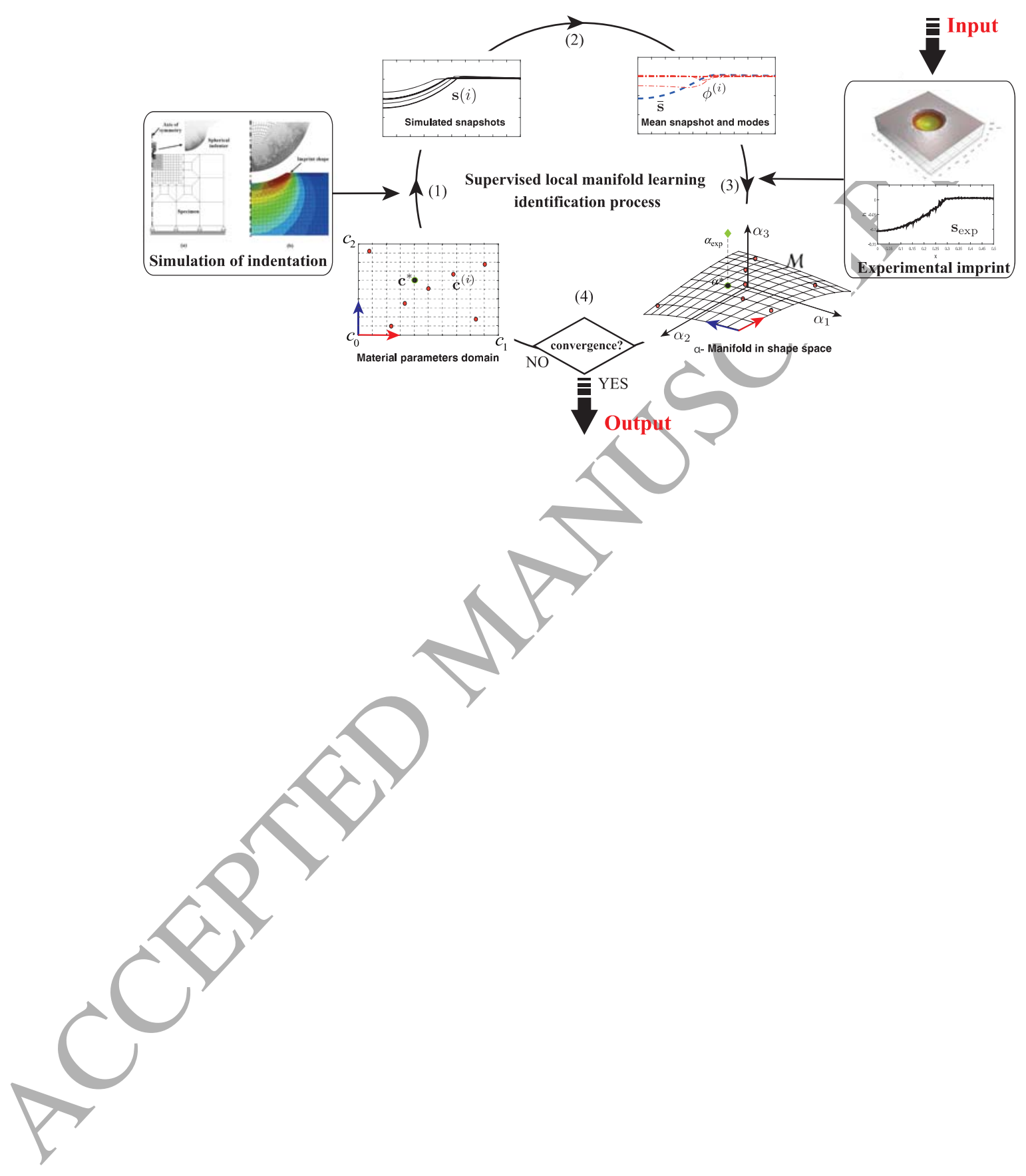




\title{
An objective meta-modeling approach for indentation-based material characterization
}

Liang Meng ${ }^{a}$, Balaji Raghavan ${ }^{b}$, Olivier Bartier ${ }^{c, 1}$, Xavier Hernot $^{c}$, Gerard Mauvoisin $^{c}$, Piotr Breitkopf ${ }^{a}$

a Sorbonne Universités, Université de Technologie de Compiègne UMR UTC-CNRS 7337, Compiègne, France

${ }^{b}$ Laboratoire de Génie Civil et Génie Mécanique EA 3913 INSA de Rennes, France

c Laboratoire de Génie Civil et Génie Mécanique EA 3913 IUT-Université de Rennes 1, France

\begin{abstract}
\end{abstract}
Over the last decade, indentation-based material characterization has shifted away from contact zone measurements for identifying the work hardening properties, and has begun to focus on inverse analysis using an experimental and simulated loading-unloading curve instead. Finite element modeling of the indenterspecimen system is a complex task and the solution to the inverse identification problem using the force-displacement curve is not necessarily unique. Also, the precise measurement of the displacement of the indenter tip requires the determination of the indenter frame compliance and indenter tip deformation. In this paper, we revisit the indentation imprint shape, using a novel approach involving image comparison by manifold learning, and show that it is able to outperform the conventional approach for a variety of cases. We illustrate our protocol using a spherical indenter on specimens of three different materials: AISI1095 steel and two aluminum alloys EN AW-2017F and EN AW-5754F.

Keywords: Indentation; Characterization; Work hardening; Plasticity; Supervised manifold learning.

\footnotetext{
${ }^{1}$ corresponding author: olivier.bartier@univ-rennes1.fr
} 


\section{Background and motivation}

The development of new materials like plastically graded materials (PGM) as well as the more recent developments in thin films exposed some of the limitations of conventional testing techniques like uniaxial tensile and compression tests. In addition, these destructive testing methods are not cost-effective since they do not allow us to reuse the test specimen. The instrumented indentation test $[1,2]$ is a popular non-destructive technique that is fastbecoming an alternative to the conventional tensile test for providing access to different mechanical properties of materials, more importantly, leading to the knowledge of their work hardening properties. The test has been successfully applied not just to metals and alloys but also ceramics [3, 4], hydrated nano-composites such as concrete [5-7], polymers [8, 9], single crystals [10] and plastically graded materials[11-17]. The primary advantages of these tests, besides their global non-destructive nature for the bulk material, include their applicability to low scale, property gradient, interface and brittle material characterizations.

The instrumented indentation is similar to a standard hardness test [18], where the hardness is determined by forcing a particular indenter into the material surface by increasing the applied load until a user-defined value is reached, after which the load is either held constant for a short duration before removal or immediately removed. The difference, compared with a hardness test, being that we continuously record the indentation load $P$ and the indenter displacement $h$ during both the loading as well as the unloading phases, generating what we call the P-h curve. More recently, after complete unloading, we can measure, analyze and post-process the residual surface of the indented zone, or indentation imprint, using a microscope with favorable accuracy. In addition, instrumented indentation may be performed accurately using depths in the nanoscale as well as in the micro- and macro- ranges [19].

The interpretation of instrumented indentation data remains a major mechanical issue, since instrumented indentation tests induce a complex state of stress in the test specimen, both compressive and tensile. For a long time, indentation 
test interpretation was essentially based on empirical/semi-empirical formulas from experimental investigations, typically measurements on the imprint. The earliest work in this regard was by Tabor [2] who characterized the deformation of a work hardening material using a representative strain, for Vickers as well as spherical indentation, which he expressed as a function of the ratio between the contact radius (of the imprint) and the indenter radius. Herbert et al [20] was among the first to directly compare instrumented indentation results with those obtained by direct tensile testing. The application of the concept of representative plastic strain $\varepsilon_{R}$ was found to significantly simplify the analysis of the indentation response and was later revisited by several authors [21-25]. The representative strain was obtained in terms of the indentation depth $h$ in place of the contact radius [26-31]. However, most of these works, in fact, all but the most recent (circa 2013 and later) did not dwell on the physical significance of the representative strain and were proposed to simplify the identification of mechanical properties using spherical indentation.

These days, the test mostly relies on exploiting the P-h curve [32-37], i.e. the continuously recorded force-displacement curve for the loading-unloading cycle. A standard deterministic identification approach is then used to minimize the cartesian distance (usually $L_{2}$ distance) between the FE simulated and measured P-h curves to obtain, for an assumed material constitutive law, the vector of material hardening parameters $\mathbf{c}^{*}$ :

$$
\begin{array}{r}
\mathbf{c}^{*}=\operatorname{Argmin}\left(J_{h}(\mathbf{c})\right), \\
J_{h}(\mathbf{c})=\operatorname{dist}\left(\mathbf{h}^{s}(\mathbf{c})-\mathbf{h}^{e}\right)
\end{array}
$$

where $\mathbf{h}$ collects instantaneous penetration depths of an indenter at time instants $i=1,2,3 \cdots N_{1}$ chosen so as to correspond to the same i.e. experimental and simulated loads on the curve; the superscript "s" referring to simulation by the method of Finite Elements (FEM), while the superscript "e" denotes "experimental". This way of determination is called "inverse analysis".

A review of the existing literature indicates that multiple authors have reported a phenomenon of identical P-h curves [38-42] being obtained for different ma- 
terials, termed as "mystical materials", which renders the solution to the inverse identification problem in Eq.(1) non-unique. In addition, this approach is plagued with myriad problems ranging from insufficient accuracy/precision [4345], the problem of indentation frame/machine compliance [45, 46] which may depend on the load $[44,47]$ or the material indented $[48,49]$, elastic deformation of the indenter tip [50], noisy input data [34, 35], difficulty in determining the exact starting point of the P-h curve $[51,52]$, bending of the specimen in thin-sheet indentation [53] and mesh-dependence [54].

A more complete and robust methodology would ostensibly involve combining the P-h indentation curve with mapping the residual deformation or indentation imprint, in an attempt to provide more reliable data for the inverse identification [11, 55-60]. However, instead of limiting ourselves to measuring the "pile-up" and "sink-in" (these terms are explained in [32]), we would need to extract maximum information from the imprint shape. Indeed, the imprint shape mainly depends on the hardness of the material but also depends on the deformation mode around the imprint, i.e the "sink in" and "pile up", which mainly depend on the hardening exponent, the yield stress and the Young modulus of the indented material [43]. The cost function in Eq. (1) may then be modified to:

$$
\begin{array}{r}
J(\mathbf{c})=J_{h}(\mathbf{c})+J_{s}(\mathbf{c}), \\
J_{s}(\mathbf{c})=\operatorname{dist}\left(\mathbf{u}^{s}(\mathbf{c})-\mathbf{u}^{e}\right)
\end{array}
$$

where $u_{j}, \mathcal{N}=1,2,3 \cdots N_{2}$ is the component of vector $\mathbf{u}$, denoting the vertical coordinate of a measured point $j$ with the initial surface of specimen as the reference plane; $N_{2}$ is the number of sample points chosen from the specimen surface, and this value depends on the resolution of the imprint scanning instrument as well as the FE mesh used.

This however does not in any way resolve the previously mentioned issues with the P-h curve, and further compound the problems present, the two sources of errors $J_{h}(\mathbf{c})$ and $J_{s}(\mathbf{c})$ are difficult to numerically compare without using a weighting function [11] which is somewhat ad hoc. All in all, the above present a solid argument in favor of using the imprint shape in place of the indentation 
curve.

Machine learning originally emerged from the fields of pattern recognition and computational learning theory, plays an increasingly important role in datadriven prediction and decision-making in this day and age of complex computational algorithms and powerful systems. Machine learning tasks are generally classified into three categories [61]: supervised, unsupervised and semisupervised. Supervised learning involves attempting to infer a relationship between the inputs and outputs of training data rather than simply clustering data based on their proximity to each other.

The recently introduced shape manifold [62-66] that has been applied successfully to a variety of material modeling problems $[67,68]$ falls into this category, and allows for a novel material parameter identification protocol based on exploiting the indentation imprint. The basic idea is building a "shape space" by applying the concept of shape manifold to describe all the imprint shapes admissible for a postulated constitutive law, combined with machine learning. This manifold is constructed by a series of simulated shape imprints, using a combination of Design of Experiments (DoE), Proper Orthogonal Decomposition (POD) [69] and manifold learning techniques.

A supervised nonlinear manifold walking algorithm was then successfully applied to spherical tip indentation tests on three different materials: (a) AISI 1095 steel, (b) EN AW-2017F and (c) EN AW-5754F aluminum alloys and the results are analyzed and discussed. The tensile curves plotted using the parameters identified by imprint mapping are compared to the experimentally obtained tensile curves for the three materials.

\section{Materials and experimental techniques}

\subsection{Materials used for test specimens}

Three materials were selected for this study: a spheroidized AISI 1095 steel, an aluminum alloy EN AW-2017F and an aluminum alloy EN AW-5754F. These materials were selected because of their fine and homogeneous microstructure 
which lead to a good reproducibility of the indentation tests.

The spheroidized AISI 1095 steel is an unalloyed steel approximately 1\% carbon that has been treated in order to transform the pearlite phase into cementite globules and ferrite, and has a body-centered-cubic (BCC) structure [70]. The standard spheroidizing annealing was carried out prior to delivery. The steel has the chemical composition shown in table 1. The microstructure of this steel,

\begin{tabular}{|c|c|c|c|c|}
\hline$C$ & $S$ & $P$ & $M n$ & $F e$ \\
\hline 1.03 & $\leq 0.05$ & $\leq 0.04$ & 0.5 & 98.8 \\
\hline
\end{tabular}

Table 1: Composition of the AISI1095 steel used in this study given in \% of weight content shown in the optical micrograph in figure 1, reveals a homogeneous and isotropic distribution of spheroidized cementite particles in a ferritic matrix.

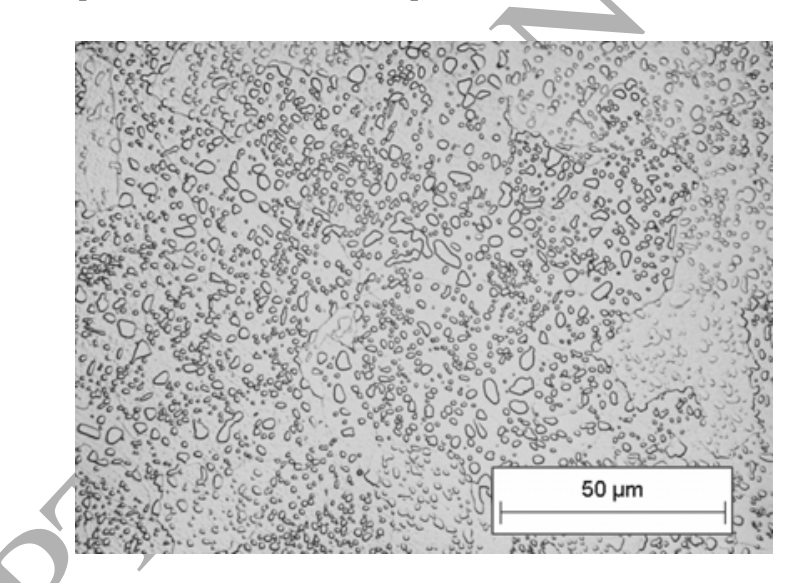

Figure 1: Microstructure of AISI1095 steel

The EN AW-2017F aluminum alloy is the second material with the chemical composition shown in table 2. For this alloy, fine particles of inter-metallic phases $\mathrm{Al}_{2} \mathrm{Cu}$ compounds oriented toward the rolled direction can be distinguished in figure 2 .

The third material was the EN AW-5754F aluminum alloy with the chemical composition shown in table 3 . This alloy is frequently used in flooring applications due to its high weldability and corrosion resistance. The microstructure 


\begin{tabular}{|c|c|c|c|c|c|}
\hline$C u$ & $M g$ & $M n$ & $S i$ & $F e$ & $A l$ \\
\hline 4.18 & 0.518 & 0.719 & 0.679 & 0.258 & balance \\
\hline
\end{tabular}

Table 2: Composition of the EN AW-2017F aluminum alloy used in this study given in \% of weight content

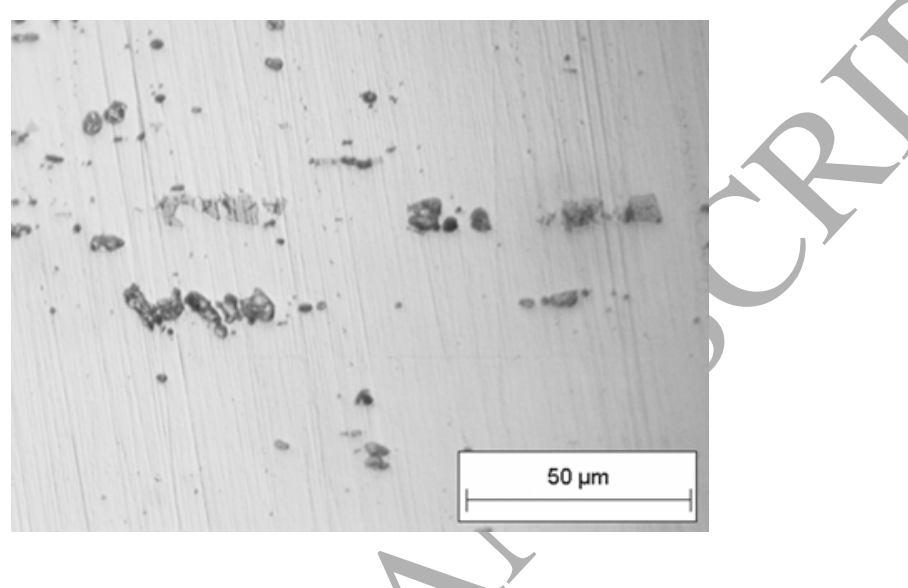

Figure 2: Microstructure of EN AW-2017F alloy

showing the size and the distribution of the $\mathrm{AlMg}_{2}$ compounds is given in figure

3.

\begin{tabular}{|c|c|c|c|}
\hline $\mathrm{SS}_{\mathrm{S}} \mathrm{Fe}$ & $M n$ & $M g$ & $A l$ \\
\hline \begin{tabular}{|l|l|}
$0.4)$ & 0.4 \\
\end{tabular} & 0.5 & 3.2 & balance \\
\hline
\end{tabular}

Table 3: Composition of the EN AW- $5754 \mathrm{~F}$ aluminum alloy used in this study given in $\%$ of weight content

\subsection{Specimen preparation}

The tensile test and indentation specimens for the $\mathrm{Al}$ alloys were carefully sectioned with a Precision Cut-Off Machine from a laminated hot rolled sheet of $6 \mathrm{~mm}$ thickness, while the steel specimen was cut from a cylindrical bar of $30 \mathrm{~mm}$ radius. This was followed by polishing using fine emery papers (up to 1200 grit) and diamond suspensions ( 6 and $3 \mu \mathrm{m}$ ) for the purpose of avoiding roughness-related uncertainties. Figure 4 shows an example of the roughness 


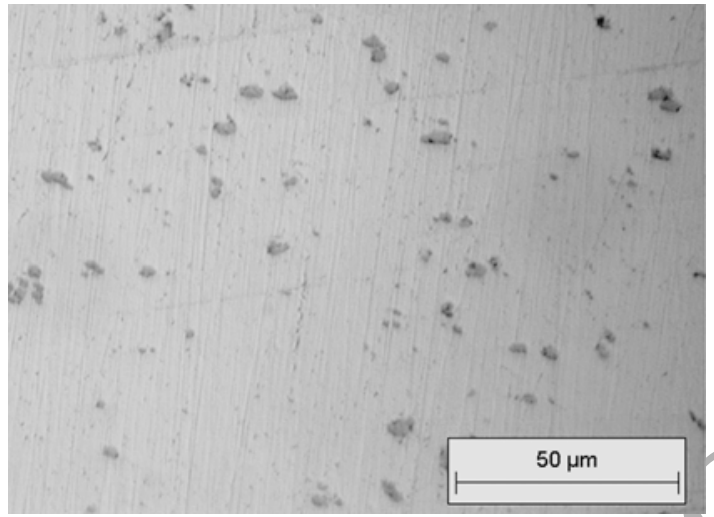

Figure 3: Microstructure of EN AW-5754F alloy

\begin{tabular}{|c|c|c|}
\hline Material & $\begin{array}{c}\text { root mean square } \\
\text { height of surface }\end{array}$ & $\begin{array}{c}\text { Arithmetic average of } \\
\text { the roughness profile }\end{array}$ \\
\hline \hline AISI1095 & $S_{q}(\mu \mathrm{m})$ ISO 25178 & $R_{a}(\mu \mathrm{m})$ ISO 4287 \\
EN AW-2017F & 0.047 & 0.021 \\
EN AW-5754F & 0.097 & 0.045 \\
\hline
\end{tabular}

Table 4: Surface roughness measures for the studied samples

profile obtained for the studied materials and Table 4 gives the values of the root mean square height of the surface $S_{q}$ obtained according the ISO 25178 standard for the studied materials.

In this table, the values of the arithmetic average of the roughness profile $R_{a}$ according the ISO 4287 standard are also provided.

\subsection{Direct tensile testing}

Tensile tests were performed on the three materials. For the aluminum alloys, the tensile behavior was studied in three different directions: $0^{\circ}$ (Rolling Direction: RD), $45^{\circ}$ (Diagonal Direction: DD) and $90^{\circ}$ (Transversal Direction: TD). The true stress-strain curves obtained until the neck for the three materials are shown in figure 5. This figure shows that because of the manufacturing 

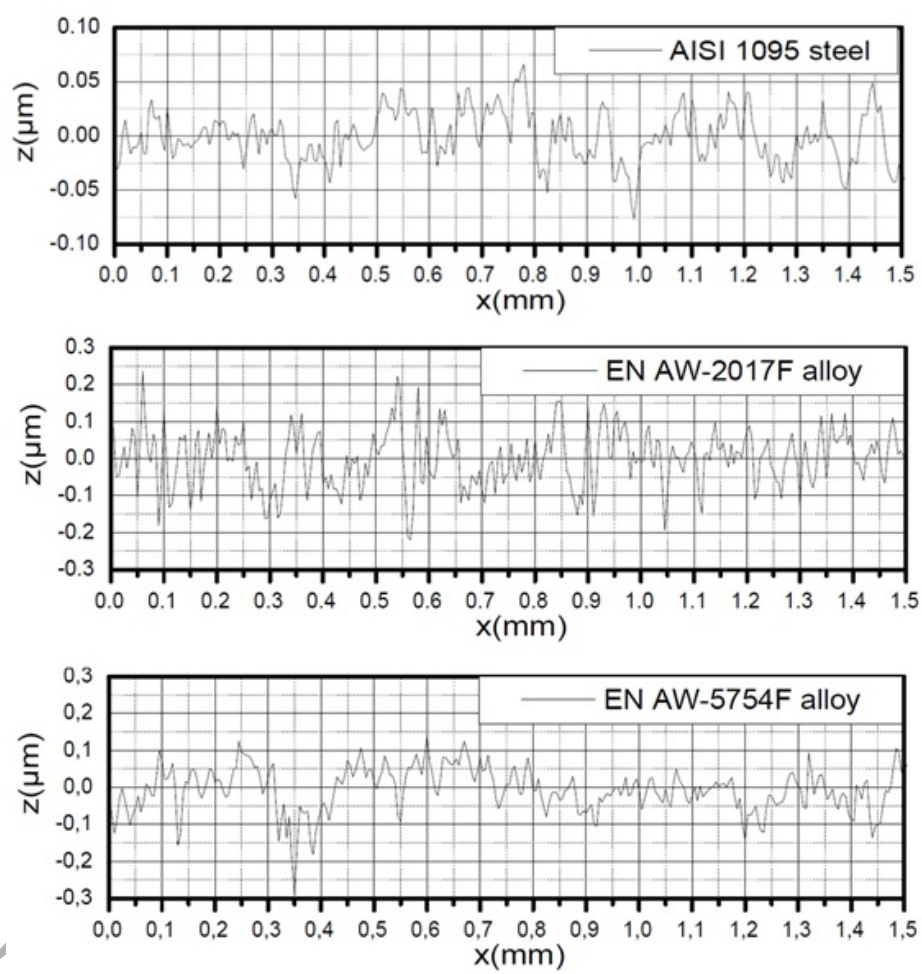

Figure 4: Surface roughness profiles for the three materials 
process, the behavior laws of the aluminum alloy are anisotropic. The elastic
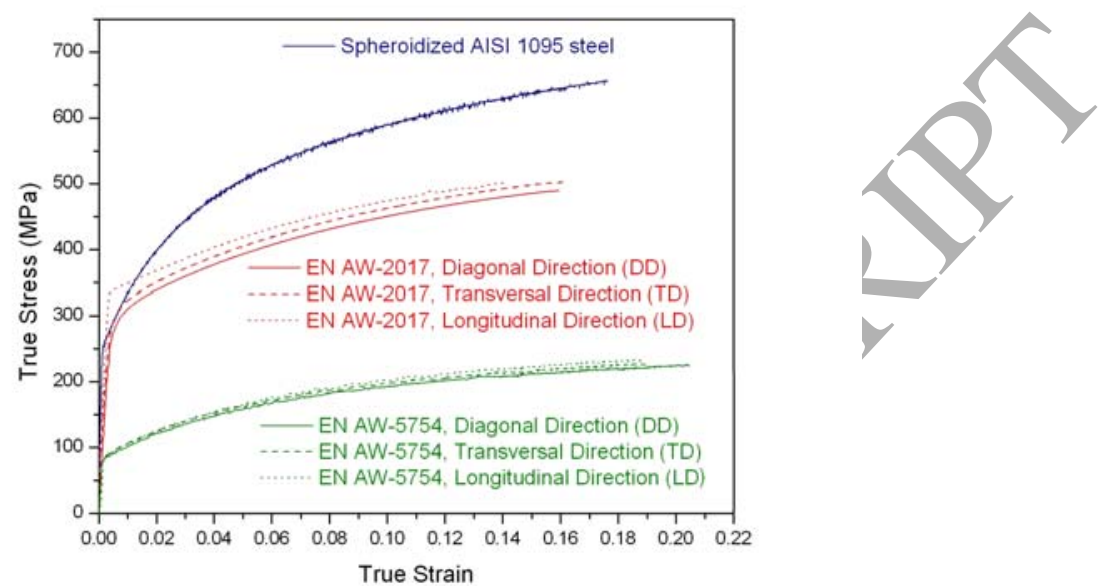

Figure 5: Tensile curves for the three materials

properties and yield strengths (determined from the tensile testing) for the three specimen materials are listed in table 5 .

\begin{tabular}{cccc}
\cline { 2 - 3 } Property & AISI 1095 & EN AW-2017F & EN AW-5754F \\
\hline Young's modulus $(E)$ & $210 \mathrm{GPa}$ & $68 \mathrm{GPa}$ & $70 \mathrm{GPa}$ \\
Poisson's ratio $(\nu)$ & 0.3 & 0.33 & 0.31 \\
Yield Strength $\left(\sigma_{y}\right)$ & $112 \mathrm{MPa}$ & $250 \mathrm{MPa}$ & $81 \mathrm{MPa}$ \\
\hline
\end{tabular}

Table 5: Elastic properties and yield strengths for the three specimen materials from tensile testing

\subsection{Indentation set-up and procedure}

Indentation tests were conducted on the three specimen samples using the setup in figure 6. We have used an industrial indenter set up composed of a tip bonded to the indenter holder (as opposed to a monoblock indenter which is a monolithic entity). The displacement was measured with capacitive sensors which were fixed near the indenter. The distance between the location of the 
sensors and the indented surface is about equal to $10 \mathrm{~mm}$. These sensors give the distance between the indenter and the indented surface of the tested material with a resolution of $0.02 \mu \mathrm{m}$.

The indenter has a spherical tip (figure 7) with radius $R=0.5 \mathrm{~mm}$ made of Tungsten Carbide with $E=600 \mathrm{GPa}$ and $\nu=0.23$ (elastic properties measured by ultrasound), but the rest of the apparatus is composed of steel. The advantage of this type of indenter is that it is easy to produce and is available for many manufacturers. The disadvantage is that the mounting of the sphere presents manufacturing difficulties, which may lead to a nonlinear load-displacement response in the assembly, thus is known to not give a very useful P-h curve on account of the presence of compound between the, indenter and the indenter holder.

Finally the distance between indents is over three times the diameter of the

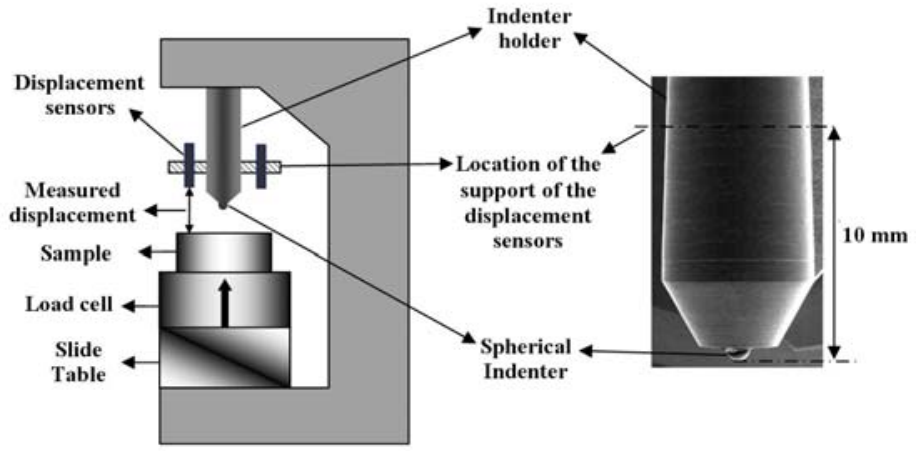

Figure 6: Experimental set up for indentation

imprint, allowing us to neglect the effects of the indents on each other.

\subsection{Analyzing the imprint shape}

The experimental imprint shape was measured using a metrological machine Altisurf 500 that works on the optical principle of chromatic confocal imaging (figure 8). The measurement was performed by using a CL2 Confocal chro- 


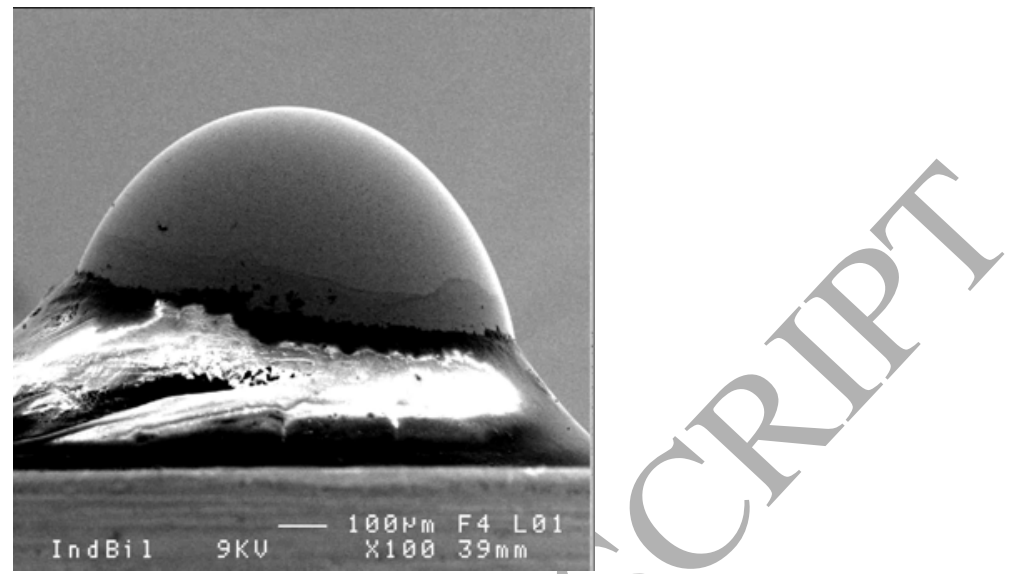

Figure 7: Indenter tip measured by a Scanning Electron Microscope(SEM)

matic probe and high-precision and motorized shifting platforms, which allow the movement of the sample being measured along the $\mathrm{X}$ and $\mathrm{Y}$ axes. The axial and lateral accuracy of the CL2 Confocal chromatic probe are $0.08 \mu \mathrm{m}$ and 1.7 $\mu \mathrm{m}$ respectively. A single imprint shape was obtained after each indentation. This shape was then pixelized using a resolution of $200 \times 200$ by scanning the vicinity of the center of the imprint. The step length along each direction was $10 \mu \mathrm{m}$ which rests with the resolution of the scanning machine.

The $3 \mathrm{D}$ imprint was then "reduced" to a $2 \mathrm{D}$ profile by using the mean profile from 4 different profiles with axes of symmetry oriented at $45^{\circ}$ with each other, after first correcting for planeness. Since in this first work, we assume isotropic behavior there is no need to consider the $3 \mathrm{D}$ imprint in this case. For an anisotropic material, we would obviously use the entire 3D imprint.

3. Constitutive model and Finite Element simulation of the indentation process

The literature surveyed indicates that FEA simulations of the instrumented indentation test have been conducted using both two-dimensional (2D) models that take advantage of simplifying factors like reduced geometrical representa- 

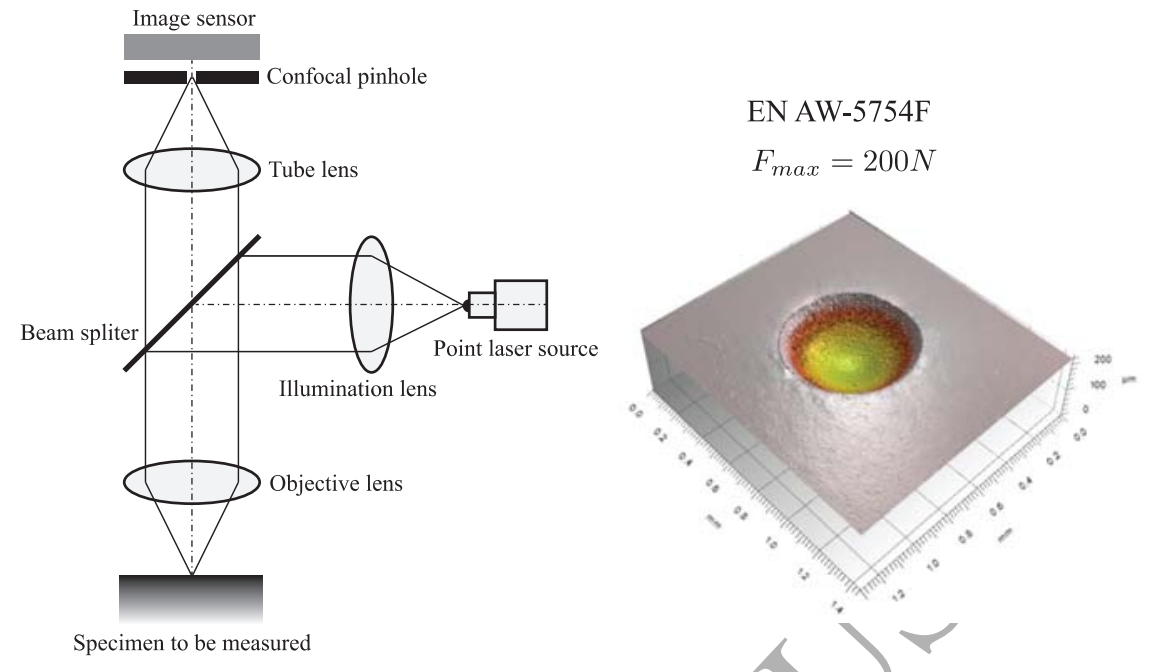

EN AW-5754F

Figure 8: Confocal laser imaging setup and a typical scanned imprint on an aluminum alloy EN AW-5754F

tions and symmetries as well as three-dimensional (3D) models[19]. With the increased abilities of modern computing stations, 3D-modeling allows the simulation of more complex geometric and material models for more realistic results. However, as shown by $[22,71,72], 3 \mathrm{D}$ models have not replaced $2 \mathrm{D}$ models since they are memory- and time-consuming, and for most stress states (e.g. biaxial) and indenter shapes (conical/spherical), we can take advantage of rotational symmetry providing that the material is isotropic. On the other hand, pyramidal (Vickers/Berkovich) indenters would benefit from 3D simulations. Since we focus on isotropic materials (with isotropic hardening) in this current paper, the uniaxial (1D) constitutive law of the indented material was taken to follow the empirical Hollomon's power law piece-wisely defined by [73, 74]:

$$
\begin{array}{r}
\sigma=E \varepsilon, \varepsilon \leq \sigma_{y} / E \\
\frac{\sigma}{\sigma_{y}}=\left(\frac{E \varepsilon}{\sigma_{y}}\right)^{n}, \varepsilon>\sigma_{y} / E
\end{array}
$$

where $\sigma$ the uniaxial stress (the flow stress in the 3D case), $\varepsilon$ is the total strain, $E$ is the elastic modulus, $\sigma_{y}$ is the yield stress and $n$ is the work hardening exponent. 
The equations for the triaxial constitutive law are presented in tensor notation for the sake of completeness:

$$
\boldsymbol{\sigma}=\boldsymbol{D}_{e}: \boldsymbol{\varepsilon}_{e}=\mathcal{K} \operatorname{tr}\left(\varepsilon_{e}\right)+2 \mathcal{G} \varepsilon_{e}^{\prime}
$$

where $\mathcal{K}=\frac{E}{3(1-2 \nu)}$ is the shear modulus, $\mathcal{G}=\frac{E}{2(1+\nu)}$ the bulk modulus, $\nu$ the Poisson ratio, $\varepsilon_{e}=\varepsilon-\varepsilon_{p}$ is the elastic (reversible) component of the strain tensor, $\boldsymbol{\varepsilon}_{e}^{\prime}$ its deviatoric component.

Eq.(3) is used to describe the flow stress with an isotropic hardening $(r)$ associated with the von Mises criterion. The yield function $f$ is then defined by:

$$
f\left(\boldsymbol{\sigma}, \sigma_{y}, \bar{\varepsilon}_{p}\right)=J(\boldsymbol{\sigma})-h\left(\bar{\varepsilon}_{p}\right)=J(\boldsymbol{\sigma})-\left\{\sigma_{y}+r\left(\bar{\varepsilon}_{p}\right)\right]
$$

where $\boldsymbol{\sigma}$ is the true (Cauchy) stress tensor, $J(\boldsymbol{\sigma})=\sqrt{3 J_{2}}, J_{2}$ being the usual second invariant of the deviatoric component $\boldsymbol{\sigma}^{\prime}$ and $h=\left(\sigma_{y}+r\right)$ is the flow stress that is assumed to increase monotonically with equivalent plastic strain $\left(\bar{\varepsilon}_{p}\right)$ :

$$
J_{2}(\boldsymbol{\sigma})=\frac{1}{2} \boldsymbol{\sigma}^{\prime}: \boldsymbol{\sigma}^{\prime} \text { where } \boldsymbol{\sigma}^{\prime}=\boldsymbol{\sigma}-\frac{1}{3} \operatorname{tr}(\boldsymbol{\sigma}) \mathbf{I}
$$

The plastic component of the strain tensor is calculated using the Prandtl-Reuss relation (associated flow rule since materials are non-porous):

$$
\dot{\varepsilon}_{p}=\dot{\lambda} \frac{\partial f}{\partial \boldsymbol{\sigma}}=\sqrt{\frac{3}{2 J_{2}}} \dot{\overline{\varepsilon_{p}}} \boldsymbol{\sigma}^{\prime}
$$

Combining these equations allows us to determine the tangent modulus (elastoplastic) tensor that is used to calculate the tangent stiffness matrix in the FE analysis

$$
\boldsymbol{D}_{E P}=\boldsymbol{D}_{e}-\frac{\boldsymbol{D}_{e}: \boldsymbol{f}_{\sigma} \otimes \boldsymbol{f}_{\sigma}: \boldsymbol{D}_{e}}{\boldsymbol{f}_{\sigma}: \boldsymbol{D}_{e}: \boldsymbol{f}_{\sigma}+H \bar{\varepsilon}_{p}}
$$

where $H$ is the derivative of the flow stress $h$ with respect to the equivalent plastic strain $\bar{\varepsilon}_{p}$ obtained from Eq.(3).

We now consider the numerical FE simulation of an axisymmetric indentation 


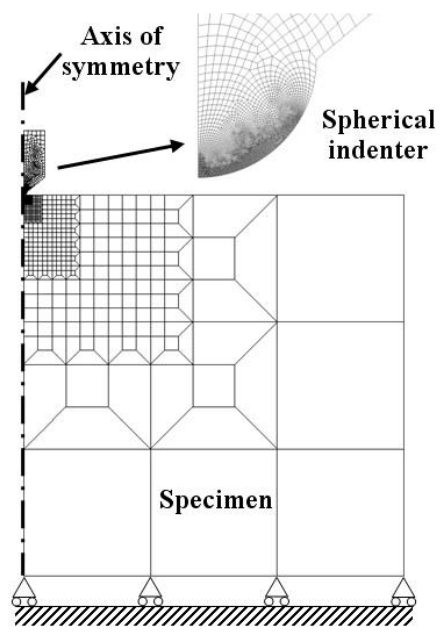

(a)

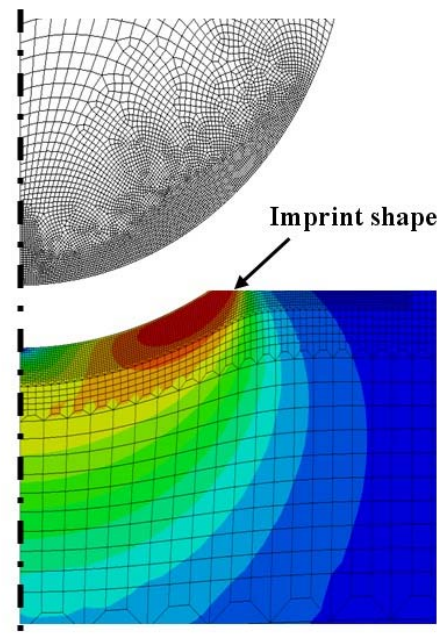

(b)

Figure 9: FE simulation model of indentation test and residual displacement of the specimen test on a specimen using a spherical tip indenter. The typical FE model and the residual deformation of the specimen are shown in figure 9. The numerical simulation of the indentation test is carried out within the commercial software ABAQUS [75] by using the FE model in figure 9 under the finite (large) strain assumption. Four-noded axisymmetric elements (CAX4) were used with 4394 elements for the specimen and 6070 elements for the indenter. The contact interface between the indenter and the specimen was characterized by a Coulomb friction coefficient of 0.1 . There is a possible dependence on the friction coefficient, which would increase with increasing maximum depth to ball radius ratio. The indenter was modeled from the indenter tip until the location where the displacement sensors were fixed on the experimental bench, and was considered as a non-rigid material with properties $600 \mathrm{GPa}$ and 0.23 for the elastic modulus and Poisson's ratio respectively. 


\section{Material characterization using the $\mathbf{P}-\mathrm{h}$ curve}

Figure 10 shows the experimental indentation curves obtained by using the spherical indenter described in the previous section, for the three materials with stress-strain curves obtained by tensile testing (figure 5).

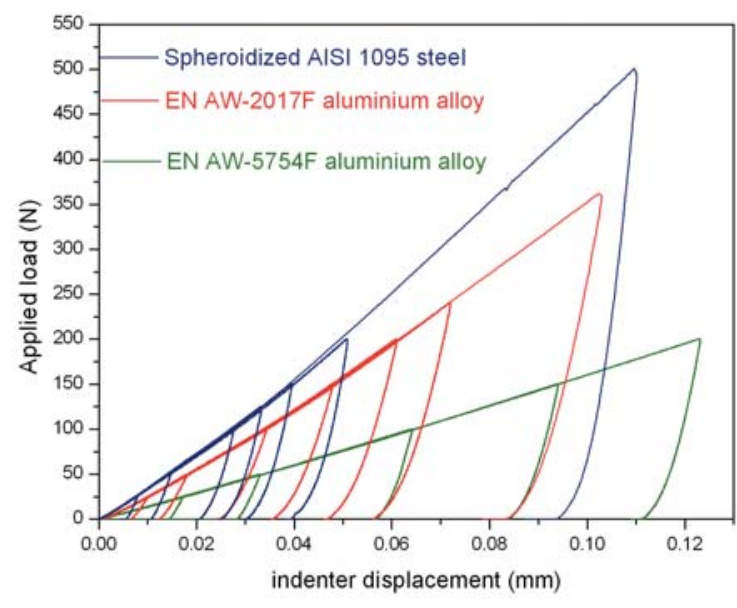

Figure 10: Experimental P-h curves for the three alloys using different maximum loads

The figure shows that for a given applied load, we obtain the highest penetration depth for the EN AW-5754 aluminum alloy and the lowest was obtained for the spheroidized AISI 1095 steel. That means that the strength of the EN AW-5754 aluminum alloy is the lowest and the strength of the spheroidized AISI 1095 steel is the highest.

As has been mentioned in the introduction, depth-sensing indentation techniques provides us with the opportunity to quantify the plastic mechanical properties of materials, including yield strength $\sigma_{y}$ and strain hardening exponent $n$. For quantification of the plastic properties using the various methodologies proposed, we need information about the variation of the indenter displacement, i.e. penetration depth of the indenter tip in the indented sample [23-28]. Since this is simply the distance between a material point of the indenter located near 
the contact and the original indented surface [15, 36, 73, 74], it was obtained using sensors, attached to the indenter holder at a distance of $10 \mathrm{~mm}$ to the indenter tip (figure 7), giving the distance between the point of attachment and the original indented surface. For our experimental bench, this corresponds then to determining the load frame compliance [43].

It is traditionally assumed that the total measured compliance $C_{T}$ corresponds to the compliance of two springs in series:

$$
C_{T}=C_{f}+C_{s}
$$

where $C_{f}$ is the load frame compliance and $C_{s}$ is the sample compliance. The total compliance $C_{T}$ is related to the total stiffness $K_{T}$ by $C_{T}=1 / K_{T}$ and corresponds to the slope of the tangent line with relation to the experimental unloading curve at the maximum loading point. The sample compliance is related to the contact stiffness $K$ by $C_{s}=1 / K$.

The contact stiffness $K$ between a spherical indenter and a flat elastic half space can be obtained by the following relationship [76-78]:

$$
K=\frac{2 \gamma E_{R} \sqrt{A_{C}}}{\sqrt{\pi}}
$$

where $A_{C}$ is the projected contact area in the plane of the indented surface, $\gamma$ is a factor introduced by [79] which depends on the elastic properties of the contact and the contact area and $E_{R}$ is the reduced modulus, calculated starting from the elastic properties of the sample $E_{s}$ and $\nu_{s}$ and of the indenter $E_{i}$ and $\nu_{i}$ such that:

$$
E_{R}=\frac{1}{\left(1-\nu_{i}^{2}\right) / E_{i}+\left(1-\nu_{s}^{2}\right) / E_{s}} .
$$

Substituting Eq.(10) into Eq.(9) leads to:

$$
C_{T}=C_{f}+\frac{\sqrt{\pi}}{2} \frac{1}{E_{R}} \frac{1}{\gamma \sqrt{A_{C}}} .
$$

Accordingly to Eq.(12), a linear representation must be obtained between the total compliance and the inverse of $\gamma \sqrt{A_{C}}$. The intercept at the origin of the straight line and the slope of this straight line lead to the values of the frame 


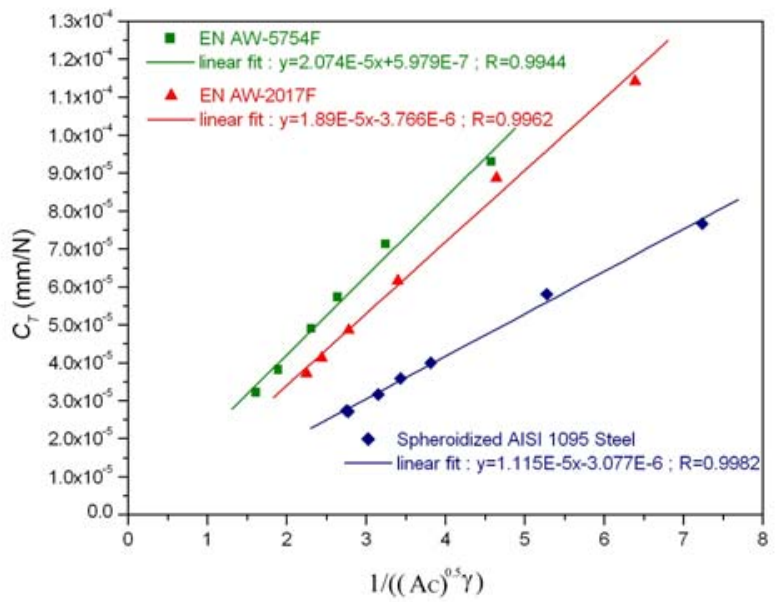

Figure 11: $C_{T}$ vs $1 / \gamma \sqrt{A_{C}}$ for the three studied materials

compliance $C_{f}$ and of the reduced modulus $E_{R}$ respectively.

From the experimental indentation tests, $C_{T}$ was calculated from the procedure adopted by Oliver and Pharr [80] The contact area $A_{C}$ was directly measured using the optical microscope after unloading. A linear regression was applied to the experimental data. Figure 11 shows the plots of the total compliance $C_{T}$ versus $1 /\left(\gamma \sqrt{A_{C}}\right)$ obtained for the studied materials. From the values of the slopes of the straight lines shown in figure 11, the reduced modulus of the contact $E_{R}$ was calculated.

\begin{tabular}{llccc}
\hline Material & $E_{R}$ & $E_{R}^{\text {exp }}$ & Error(\%) \\
\hline AISI1095 & $169,152 \mathrm{MPa}$ & $74,692 \mathrm{MPa}$ & -56 \\
EN AW-2017F & $68,106 \mathrm{MPa}$ & $47,070 \mathrm{MPa}$ & -33 \\
EN AW-5654W & $69,007 \mathrm{MPa}$ & $42,921 \mathrm{MPa}$ & -39 \\
\hline
\end{tabular}

Table 6: Experimental and theoretical reduced elastic modulii

Table 6 compares the theoretical and experimental reduced modulii $\left(E_{R}\right.$ and $E_{R}^{e x p}$ respectively). The table shows that big differences between the experimental and theoretical results were obtained. The experimental reduced moduli 
are much lower than the theoretical ones. In addition, figure 11 also shows that $C_{f}$, which is the intercept at the origin of the straight line, takes negative values for the spheroidized Steel and the EN AW-2017F aluminum alloy. However, it should take a positive value since it represents the compliance of the holder of the sphere. Previous work showed that $C_{f}$ was about equal to $3.2 \times 10^{-6} \mathrm{~mm} / \mathrm{N}$ in the case of a similar indenter, which was made of a strongly crimped carbide ball in a steel holder [43].

These results demonstrate that the model of two springs in series proposed to depict the total compliance of an instrumented bench is not valid when using the spherical indenter setup shown in figures 6 and 7 . The reason is certainly attributable to a bad crimping of the carbide ball in the holder and/or presence of compound between the carbide ball and the hólder which lead to nonlinearities of displacement at the contact zone between this ball and this holder. The holding system can not be associated with a linear spring.

Because of these nonlinearities and the drastic reduction in reduced modulus, the indentation curves can hardly be used to determine the work hardening laws of the studied materials from the proposed methods in the literature [15, $26-$ $31,36]$. That is the reason why we propose using the imprint shape in place of the indentation curve in the following sections.

\section{Material characterization by shape manifold-based image correla- tion using the indentation imprint}

We first performed FE simulations of an axisymmetric indentation test on the three alloys with a spherical tip indenter of $0.5 \mathrm{~mm}$ radius, as described in section 3 .

\subsection{Imprint shape}

The simulated imprint shape obtained using the previously detailed FE model is represented by a set of vertical displacements of the surface nodes collected in vector $\mathbf{s} \in \mathcal{R}^{D}$, where $D$ is the number of surface nodes of the mesh 
in the zone of interest. Due to the axisymmetric property of the model, the simulated imprint shape was obtained by extracting the vertical displacements of sample points on half of the imprint. We note that $D \geq 400$ for the examples we have studied.

\subsection{Design of Experiments and POD}

We need to construct a reduced order basis for 2 main reasons:

1. The large number of nodal displacements needed for an accurate description of the imprint shape. Even for a $1 \mathrm{D}$ imprint (as in the case of this paper) we require over 500 surface nodes.

2. We need to find an appropriate vector space where we can characterize the discrepancy in imprint shapes as a function of the vector set of material hardening parameters.

As explained in the introduction, we use the shape manifold protocol to develop this reduced basis vector space and perform the identification within. The fundamental hypothesis of the shape manifold approach applied to the imprint mapping problem is that the set of all possible imprint shapes obtainable by FE simulation using the assumed constitutive law (in our case the power law), forms a smooth manifold in an $m$-dimensional space $(m \ll D)$, where we will perform the identification.

The $M$ numerical experiments for the varying set of $\mathbf{c}^{(i)}, i=1,2 \cdots M$ (generated by Latin Hypercube Sampling (LHS) around $\mathbf{c}^{\prime}$ ) yield a set of imprint shapes $\mathbf{s}^{(i)}=\mathbf{s}\left(\mathbf{c}^{(i)}\right)$ obtained by FE simulation, and these are our imprint snapshots (figure 12). Singular value decomposition of the matrix of snapshots $\left[\mathbf{s}^{(1)}, \mathbf{s}^{(2)}, \ldots \mathbf{s}^{(M)}\right]$ yields the basis $\Phi$. Each snapshot $\mathbf{s}^{(i)}$ can then be accurately reconstructed by

$$
\mathbf{s}^{(i)}=\overline{\mathbf{s}}+\boldsymbol{\Phi} \boldsymbol{\alpha}^{(i)}=\overline{\mathbf{s}}+\sum_{j=1}^{M} \alpha_{j}^{(i)} \phi_{j},
$$

where $\overline{\mathbf{s}}$ is the mean snapshot (constant over the neighborhood) and $\alpha_{j}^{(i)}$ is the projection coefficient for the $i^{\text {th }}$ snapshot on the $j^{\text {th }}$ mode $\phi_{j}$, the projection 
coefficients vary with the material parameters.

$$
\boldsymbol{\alpha}_{j}^{(i)}=\phi_{j}^{\mathrm{T}}\left(\mathbf{s}^{(i)}-\overline{\mathbf{s}}\right), j=1,2 \cdots M
$$

Figure 12 gives the mean $\overline{\mathbf{s}}$ of all snapshots $\mathbf{s}^{(i)}$ as well as the modes scaled by

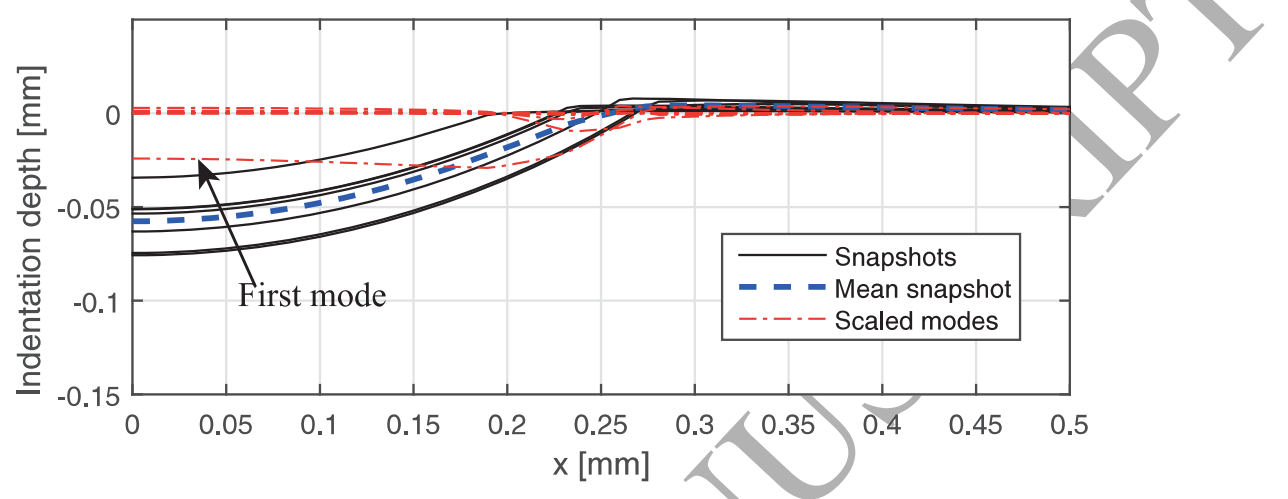

Figure 12: Imprint snapshots for varying material parameters, mean snapshot, and POD modes scaled by corresponding eigenvalues

the corresponding eigenvalues. The scaled modes demonstrate that, except the most significant mode (the first mode), other modes mainly contribute to the pile-up/sink-in zone.

\subsection{Manifold $\mathcal{M}$ of admissible imprint shapes}

For the $M$ simulated imprint snapshots, the coordinates in $\alpha$-space are given by Eq. (14) By our fundamental assumption, all imprint shapes admissible with a given material law lie on a smooth manifold

$$
\mathcal{M}(\boldsymbol{\alpha})=0 .
$$

We use a parametric representation of $\mathcal{M}$

$$
\alpha_{j}(\mathbf{c})=\mathbf{p}^{\mathbf{T}}(\mathbf{c}) \mathbf{a}^{(j)}, j=1,2 \cdots M
$$

with polynomial basis $\mathbf{p}$ and the coefficient vectors $\mathbf{a}^{(j)}$ approximated for all $\boldsymbol{\alpha}_{j}^{(i)}, i=1,2 \cdots M$ by minimizing the moving least-square error

$$
\mathbf{a}^{(j)}=\operatorname{Argmin} \frac{1}{2} \sum_{i=1}^{M} w\left(\mathbf{c}^{(i)}, \mathbf{c}\right)\left(\mathbf{p}^{\mathbf{T}}\left(\mathbf{c}^{(i)}\right) \mathbf{a}^{(j)}-\boldsymbol{\alpha}_{j}^{(i)}\right)^{2} .
$$




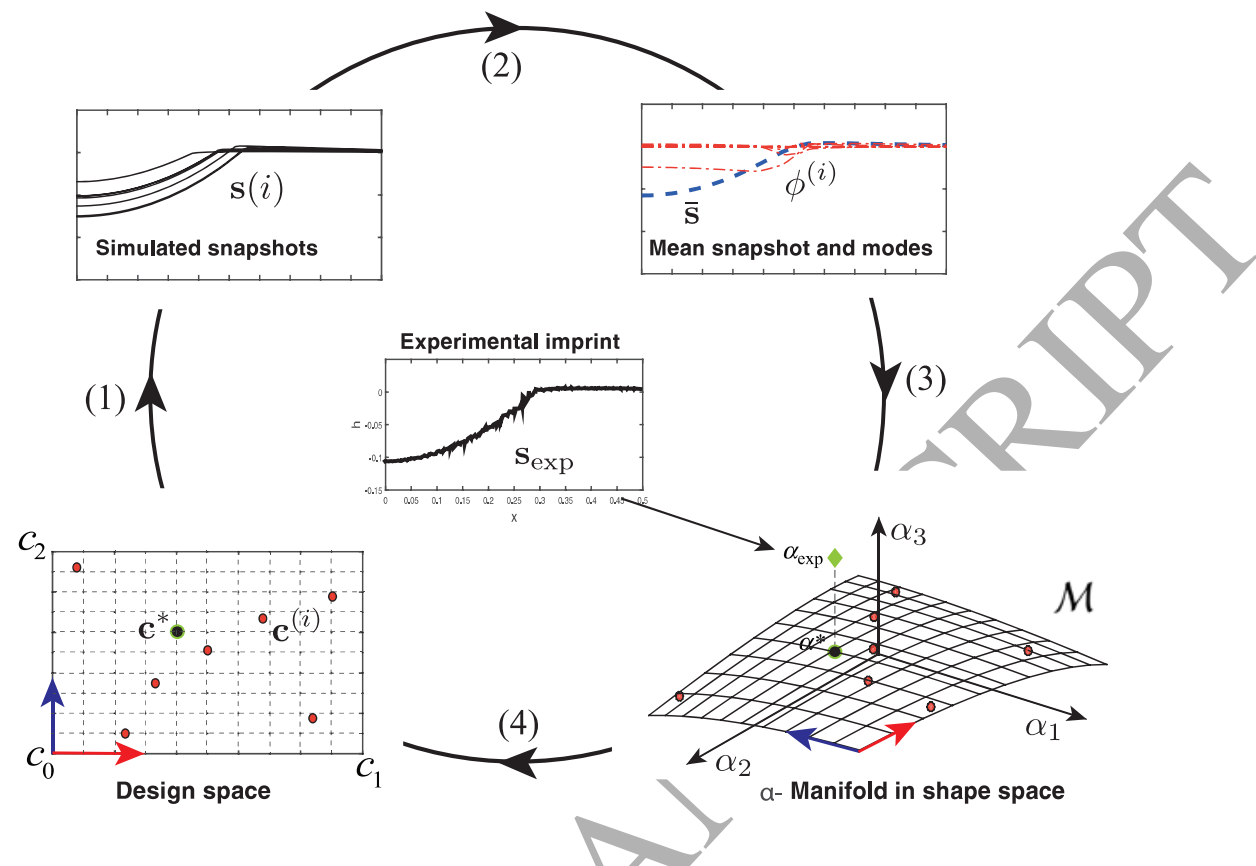

Figure 13: Identification using correspondence between design space and $\alpha$-space

Thus a one-to-one relationship is built up between the design space and the shape manifold in $\alpha$-space:

1. For each point in the design space i.e. set of parameter values, there exists a corresponding point on the manifold according to Eq.(16).

2. Each point on the manifold corresponds to an imprint snapshot and, thus a set of parameter values.

On the other hand, the experimental imprint is employed without any denoising preprocessing, and its representation in manifold space $\alpha_{\exp }$ is derived from Eq. (14). However, it does not necessarily lie exactly on the constructed manifold due to the presence of measurement error and manifold inaccuracy.

Considering all these, we proposed an identification procedure as illustrated in figure 13 (only a 3D section of the higher-dimensional space is shown for visualization). The experimental imprint is mapped into the shape space constructed by a series of simulated imprints, and is projected onto the constrained mani- 
fold to yield an estimate of the parameter set. For saving computing time, local manifold of lower order should be constructed piece by piece to approximate the geodetic hyper manifold.

\subsection{Manifold Learning algorithms}

In [64], the authors have proposed three manifold learning algorithms: zooming, panning and the combined panning/zooming. These algorithms, in partic ular the third, have outstanding capacity to guide the iterative convergence during the identification procedure. However they do have certain limitations, i.e., low accuracy, slow convergence and complex programming. Even with the combined panning/zooming method [64], switching from one approach to the other is not a trivial issue. Moreover, the glaring limitations of the zooming algorithm can never be completely avoided, meaning that we always risk missing the optimal solution since the search zone is explored using a dichotomizing strategy.
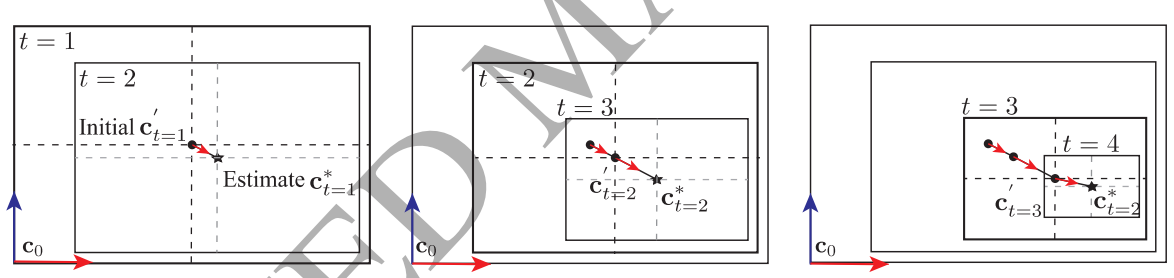

Figure 14: Floating search strategy

Therefore, in the present work, we propose a modified zooming method, with which the already-searched space could be searched again in order to make up for the bad-estimation from the previous step. In this algorithm, first of all, we use a user-defined multiplier $\beta$, namely the shrinking ratio of design space after iteration, that is designed to control the convergence of the identification procedure. Secondly, and this is more important, each searching window needs to be centered on $\mathbf{c}^{\prime}$, which is also the estimate from last iteration $\mathbf{c}^{*}$, making it possible to cover a portion of the design space that has already been searched.

The pseudo time of the identification, i.e., the iteration step number, is indexed 
by $t$.

The present algorithm has been validated using the three test cases in the present work, and has proved to be stable regardless of the complexity of the problem and the desired accuracy. We have named this algorithm a "Floating search" and the search pattern is shown in figure 14. Quite obviously in the figure, $\mathbf{c}_{t=1}$ is equal to $\mathbf{c}_{t=2}^{\prime}$

\subsection{Results obtained after inverse analysis and discussion:}

The methodology proposed in the previous section was applied to an axisymmetric indentation test on (see table 5):

1. AISI1095 steel

2. EN AW-2017F and

3. EN AW-5754F aluminum alloys using the spherical tip indenter shown in figure 7 with a radius of $0.5 \mathrm{~mm}$. The experimental (real) imprint shape was obtained using the Altisurf 500 machine described in the previous section.

From Eq. (2) and following the convention [81], the Hollomon power law allows us to describe the hardening behavior law of the material using the two material parameters to be identified : yield stress $\sigma_{y}$ and strain hardening exponent $n$. Even if we already have a general estimation for the yield stresses of the above-mentioned materials, the parameters are identified in a large design space so as to ensure the accuracy during the inverse analysis and allow to test for robustness.

The numerical simulation of the loading and unloading phases of the indentation test is performed with ABAQUS by using the FE model in figure 9 . The maximum values of the indentation force $F_{\max }$ are $500 \mathrm{~N}, 360 \mathrm{~N}$ and $200 \mathrm{~N}$, respectively for the three studied materials. The reason these particular maximum loads were chosen has to do with obtaining imprints of similar size for the 3 materials $\left(h_{\max } / R \approx 0.2\right)$. This ratio is very important since it has been shown that a sufficient accuracy of the obtained results is obtained only for a 
sufficiently high $h_{\max } / R$ ratio (when using the loading-unloading curve). On the other hand, for extremely high values of this ratio, the results obtained have a greater sensitivity to the choice of friction coefficient. The value chosen in this work presents a good compromise.

A reasonable argument could be made for using the same load across all materials (i.e. load controlled experiment) in place of our depth controlled experiment (similar size imprints). The issue of course is that the same load applied to different materials (with different yield limits and hardening parameters) may or may not result in plastic deformation for all the materials (thus yielding an imprint which is necessary for using our approach). Also, while not directly related to this issue, it has been demonstrated that the identifiability of material parameters depends on whether the manifold is generated using a load controlled design of experiments (DoE) or a depth/penetration controlled DoE [82], in other words, the difficulty of inverse identification of material parameters may be at least partly related to the implementation of indentation test. [82] states that "depth controlled" in FE simulation provides us with a slightly better-conditioned problem in comparison with "load controlled" ones. A quadric polynomial basis (2D), containing 6 different terms, was applied for the construction of the smooth manifold. 7 snapshots were chosen in each DoE using Latin Hypercube sampling [83]. As earlier, the SVD was performed with a full basis of size 7 without truncation. The parameters were identified in this mathematical space by using the criterion in Eq. (18):

$$
\mathbf{c}^{*}=\operatorname{Argmin}\left\|\left(\boldsymbol{\alpha}_{\exp }-\boldsymbol{\alpha}(\mathbf{c})\right)\right\|, \boldsymbol{\alpha}(\mathbf{c}) \in \mathcal{M} .
$$

The convergence patterns of material parameters identified for the three studied specimens by the proposed floating manifold search algorithm are compared in figure 15. It is fairly clear that the "floating search" approach gives a good and steady convergence of both plastic parameters as well as the criterion in (18). Other possibilities, including the use of a greater number of snapshots with a quadratic basis or a lower-order polynomial basis were also validated [64]. The identified power-law parameters are summarized in table 7 . 

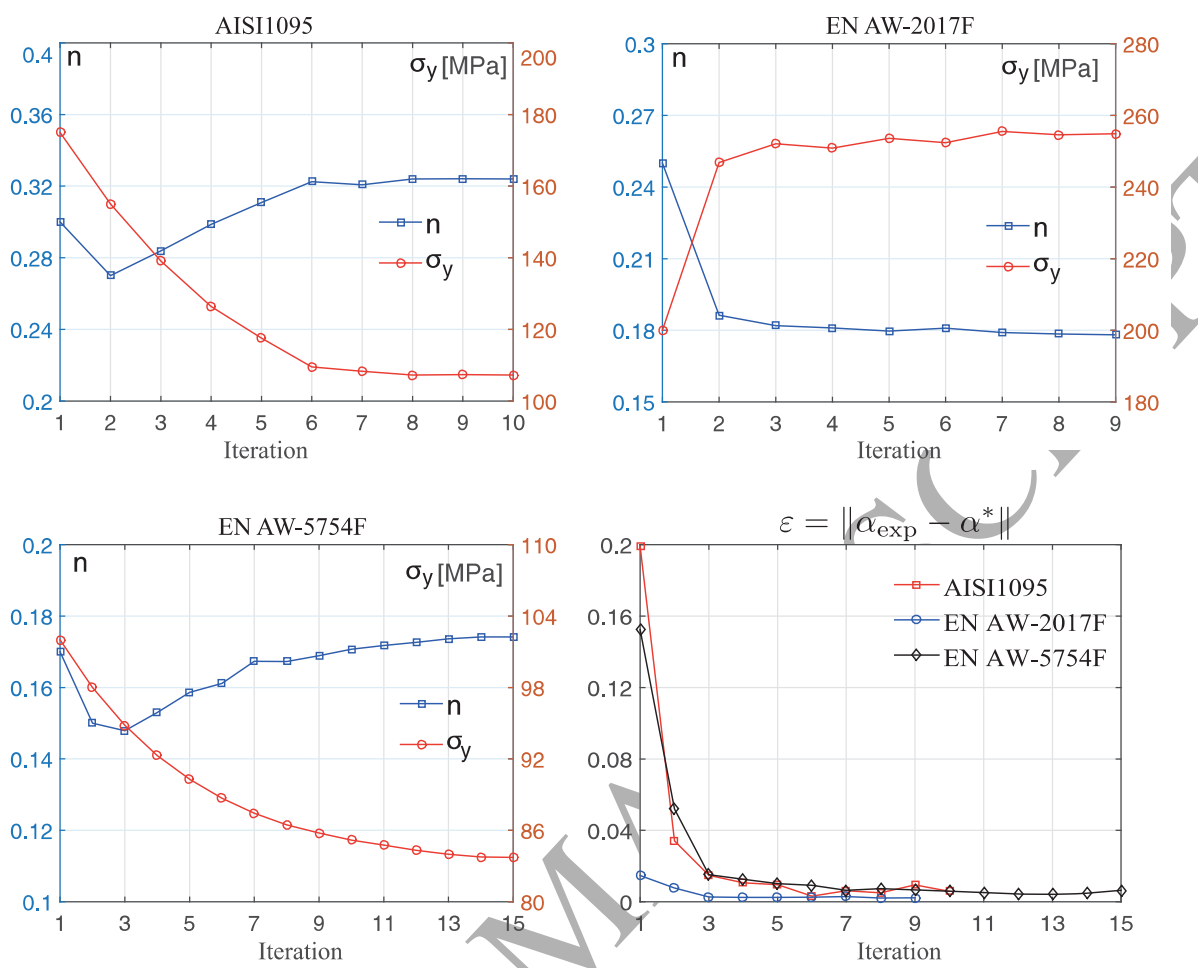

Figure 15: Convergence patterns for the parametric identification of $n$ and $\sigma_{y}$, and error $\varepsilon$ for three studied materials

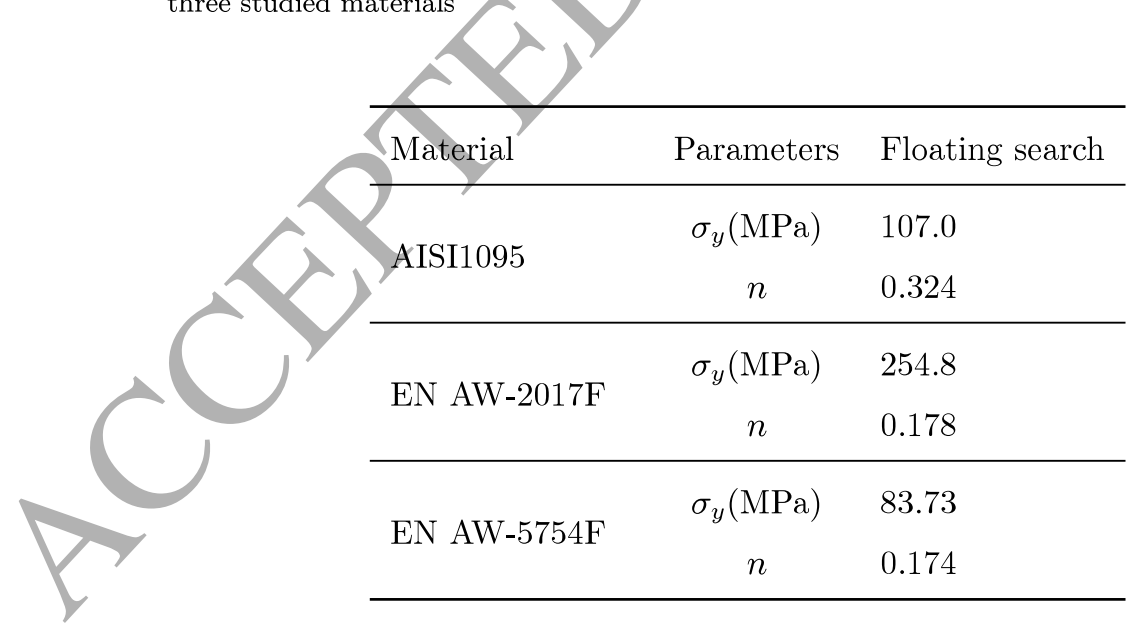

Table 7: Summary of identified hardening parameters for the three materials, where $\sigma_{y}$ is the yield strength and $n$ is the strain-hardening exponent. 
In figure 16, EN AW-2017F alloy was chosen so as to demonstrate that local manifold decrease in size along with the iterations, since a shrinking multiplier $\beta=0.8$ was chosen for the floating search protocol. Moreover, we also noticed that the new estimate of each iteration converge towards the origin of the coordinate system simply due to the reason that all the snapshots were centered before SVD. Similar results were also obtained for other two materials, thus were omitted here.

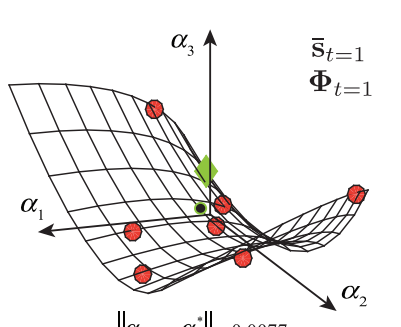

$\left\|\alpha_{\text {cxp }}-\alpha^{*}\right\|=0.0077$

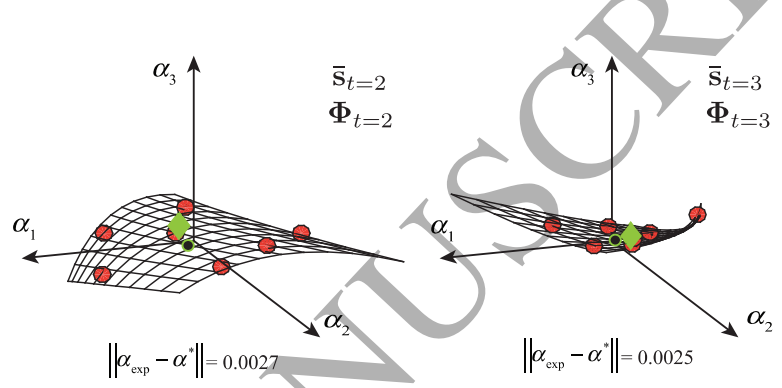

Figure 16: Local manifold convergence for EN AW-2017F

The identified imprint shapes using the supervised manifold learning algorithm are shown in figure 17 for AISI1095 (along with the imprint snapshots of each iteration), and in figure 18 for the two aluminum alloys.
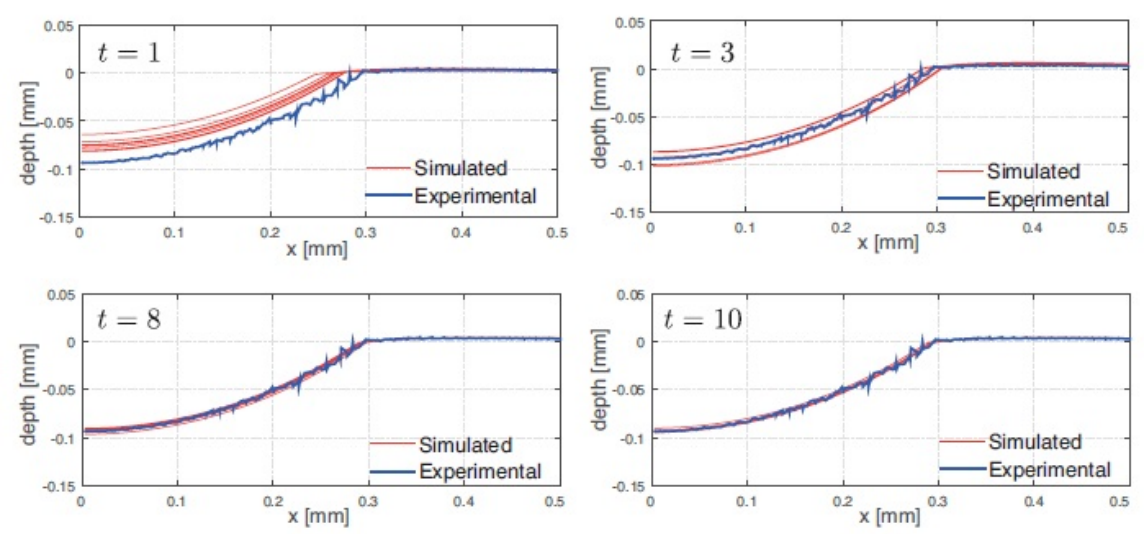

Figure 17: Iterative convergence of imprint shape for AISI1095.

Finally, the stress-strain curves plotted by employing the identified material 

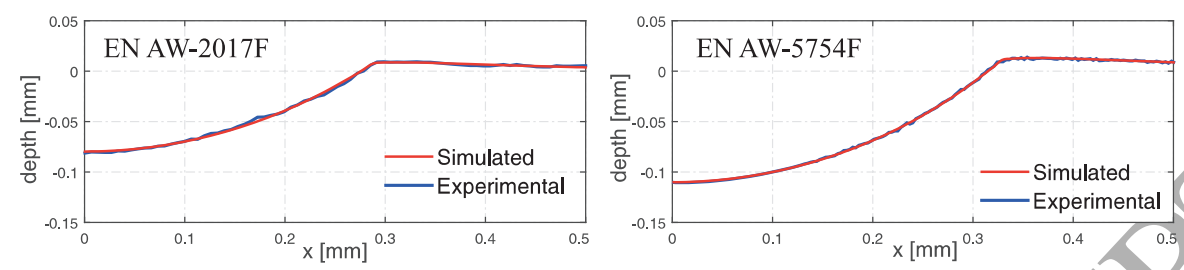

Figure 18: Final converged imprint shapes for EN AW-2017F and EN AW-5754F

Figure 19: Tensile loading curve using identified power law parameters for AISI1095 steel

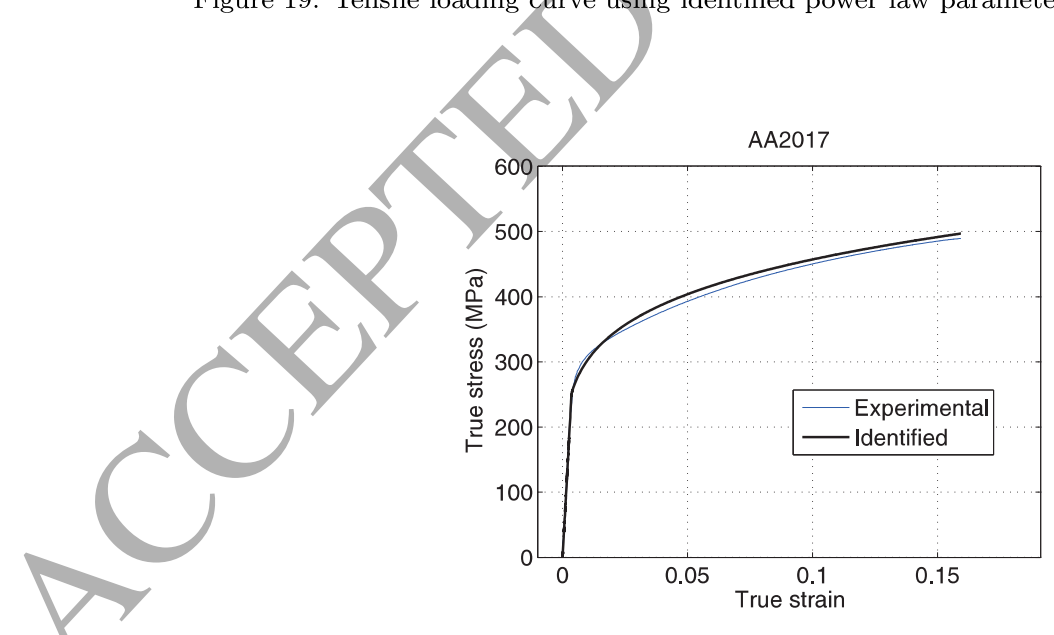

Figure 20: Tensile loading curve using identified power law parameters for EN AW-2017F alloy 


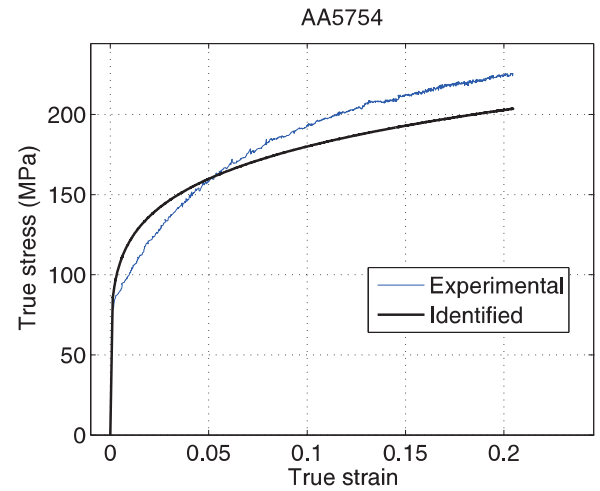

Figure 21: Tensile loading curve using identified power law parameters for EN AW-5754F alloy

properties were compared with those by uniaxial tensile test in figures 19, 20 and 21 .

\subsection{Comments and perspectives}

Figures 17 and 18 show that the manifold learning approach is able to iteratively reduce the distance between the simulated and experimental imprint shapes in order to elegantly identify the material hardening parameters, and that the identified (simulated) imprint correlates very well with the experimental. Furthermore, the POD-based approach appears to be a "physics-based" smoothing operation on the experimental (noisy) imprint.

That said, when we use the identified sets of hardening parameters in the aforementioned power-law expression in an attempt to "recreate" the experimental tensile hardening curves for the 3 materials previously seen in figure 5 , we don't always see the same degree of success. From figure 20, we get a good fit between the two sets of tensile curves indicating that the material appears to follow an isotropic "power law" hardening, the material is probably isotropic and homogenous, the yield surface is given by the von Mises criterion, etc, leading to a single indentation test being able to reproduce the tensile curve with sufficient precision.

On the other hand, figures 19 and 21 see a gap between the tensile curves despite 
being able to obtain a near perfect correlation between the imprints and a very good estimation of yield stress $\sigma_{y}$ (table 8 ). We may be tempted to chalk this up to the indentation size effect (ISE) and the heterogenity of the specimen but if we look at the microstructures of the materials characterized and estimate the size of the heterogeneities $(<10$ microns from the figures in section 2.1) with respect to the radius of the indenter tip $(0.5 \mathrm{~mm})$ or even the size of the imprints obtained (in the current section), we see that the ratio is very low, allowing us to assume an almost homogeneous specimen being indented for the cases we have identified.

\begin{tabular}{|c|c|c|}
\hline Material & AISI1095 & EN AW-2017F EN AW-5754F \\
\hline$\sigma_{y}(\mathrm{MPa})$ by indentation & 107.0 & 83.73 \\
\hline$\sigma_{y}(\mathrm{MPa})$ by tensile & 112 & 250 \\
\hline Relative error & $4.46 \%$ & $3.37 \%$ \\
\hline
\end{tabular}

Table 8: Comparison of identified yield stresses $\sigma_{y}$ for the three materials.

In addition, we verify the observation made in the literature regarding a higher value of the exponent $n$ corresponding to lower values of the "pile up" in the imprint (table 9). Clearly, the obtained sets of results and experimental observations in the literature are highly coherent.

\begin{tabular}{lclc}
\hline Material & AISI1095 & EN AW-2017F & EN AW-5754F \\
\hline$n$ & 0.324 & 0.178 & 0.174 \\
\hline$h_{c}(\mu m)$ & 4.7 & 10.6 & 13.7 \\
\hline
\end{tabular}

Table 9: Correlation between strain hardening exponent and "pile up" measured on the experimental imprint.

Basically, we must remember that tensile testing is a more "global" method while indentation is a "local" method. Even if the microstructure appears homogenous, we could have a non uniform evolution of residual stresses ergo hardening behavior, and indentation at different points will (as is usually the case) give different results. In addition, the material itself may not necessarily be 
homogenous and isotropic, and may or may not follow an isotropic hardening, not to mention, there is no guarantee that the power law assumption is valid for the materials chosen. This means that indentation based identification may or may not give the same result as tensile testing.

Finally, the phenomenon of the indentation-identified stress-strain curve(s) intersecting the tensile identified curve around the representative strain has already been shown in the literature $[14,15]$.

An obvious issue with the imprint only approach is the need for prior identification of the elastic parameters, for which using the unloading portion of the $\mathrm{P}-\mathrm{h}$ curve is necessary, unless prior information about the material is available. As far as the problem of unicity is involved, using the imprint alone may or may not necessarily lead to a unique solution. A statistical analysis using several different initial estimates for the manifold learning algorithm could verify the unicity or lack thereof for the solutions obtained, but has not been performed in this work. That said, it makes sense that the addition of the loading-unloading curve to the manifold learning approach is likely to help us narrow down a unique solution. In addition, multiple-depth indentations at the same point, as well as indentations at multiple locations, using the imprint-based protocol, are areas currently under investigation. The next step though will involve using a more advanced behavior law and the use of a 3D imprint.

\section{Conclusions}

The last few years have revealed a steady shift away from imprint measurement towards post-processing the loading-unloading curve using inverse analysis in indentation-based characterization of the plastic parameters of a material, despite the myriad problems associated with using this approach with a spherical indenter. This is ostensibly due to the difficulties associated with adequate imprint mapping.

In this paper, the authors proposed a return to the imprint shape for indentationbased characterization using a complete manifold learning protocol that at- 
tempts to circumvent the usual problems related to the loading-unloading curve by relying solely on imprint mapping. The traditional approach was replaced by a nonlinear supervised manifold learning-based image comparison protocol. The material parameters obtained using the proposed approach were compared with the corresponding values identified by direct tensile testing. The traditional inverse approach using the loading-unloading curve was replaced by this imprint mapping approach for indentation using a spherical indenter on a variety of materials. The results indicate that the proposed imprint mapping protocol performs favorably and gives comparable results to the direct tensile testing approach.

A major advantage of using the approach is the ability to map imprints in three dimensions without any real change in the implementation, thus potentially allowing us to characterize anisotropic materials using the same exact protocol with a more complex constitutive law. A logical extension of the protocol is to enrich the imprint information with the $\mathrm{P}$-h curve for even more accurate identification and potentially resolve the problem of non unicity in the identification problem.

\section{Acknowledgements}

This study is art of a project funded by the National Natural Science Foundation of China (Ref No. 11620101002) and the China scholarship Council (Ref No. 201404490062).

\section{References}

[1] B. W. Mott, Micro-indentation hardness testing, Butterworths Scientific Publications, 1956.

[2] D. Tabor, The hardness of metals, Vol. 10, ClarendonP, 1951.

[3] A. Mukhopadhyay, S. Datta, D. Chakraborty, On the microhardness of silicon nitride and sialon ceramics, Journal of the European Ceramic Society 6 (5) (1990) 303 - 311. 
[4] A. Mukhopadhyay, Comparative study of indentation fatigue in structural ceramics, Journal of Materials Science Letters 18 (4) (1999) 333-337.

[5] G. Constantinides, F.-J. Ulm, K. Van Vliet, On the use of nanoindentation for cementitious materials, Materials and Structures 36 (3) (2003) 191-196.

[6] F.-J. Ulm, M. Vandamme, C. Bobko, J. Alberto Ortega, K. Tai, C, Ortiz, Statistical indentation techniques for hydrated nanocomposites: Concrete, bone, and shale, Journal of the American Ceramic Society 90 (9) (2007) 2677-2692.

[7] S. Kamali-Bernard, D. Keinde, F. Bernard, Effect of aggregate type on the concrete matrix/aggregates interface and/its influence on the overall mechanical behavior. a numerical study, Key Engineering Materials 617 (2014) $14-17$.

[8] V. Tvergaard, A. Needleman, Polymer indentation: Numerical analysis and comparison with a spherical cavity model, Journal of the Mechanics and Physics of Solids 59 (9) (2011) $1669-1684$.

[9] Y. Lu, D. Shinozaki, Effects of substrate constraint on micro-indentation testing of polymer coatings, Materials Science and Engineering: A 396 (1-2) (2005) $77-86$.

[10] P. A. Sabnis, S. Forest, N. K. Arakere, V. A. Yastrebov, Crystal plasticity analysis of cylindrical indentation on a ni-base single crystal superalloy, International Journal of Plasticity 51 (2013) $200-217$.

[11] C. K. Moy, M. Bocciarelli, S. P. Ringer, G. Ranzi, Identification of the material properties of al 2024 alloy by means of inverse analysis and indentation tests, Materials Science and Engineering: A 529 (2011) 119-130.

[12] R. C. Paietta, S. E. Campbell, V. L. Ferguson, Influences of spherical tip radius, contact depth, and contact area on nanoindentation properties of bone, Journal of biomechanics 44 (2) (2011) 285-290. 
[13] R. F. Gibson, A review of recent research on nanoindentation of polymer composites and their constituents, Composites Science and Technology 105 (2014) 51-65.

[14] C. Moussa, X. Hernot, O. Bartier, G. Delattre, G. Mauvoisin, Identification of the hardening law of materials with spherical indentation using the average representative strain for several penetration depths, Materials Science and Engineering: A 606 (2014) 409-416.

[15] C. Moussa, O. Bartier, G. Mauvoisin, X. Hernot, J.-M. Collin, G.J Delattre, Experimental and numerical investigation on carbonitrided steel characterization with spherical indentation, Surface and Coatings Technology 258 (0) (2014) $782-789$.

[16] M. Sebastiani, A. Cusmà, E. Bemporad, F Carassiti, Elastic anisotropy of coatings by AFM analysis of microindentations, Surface Engineering 30 (1) (2014) 41-47.

[17] H.-Y. Amanieu, D. Rosato, M. Sebastiani, F. Massimi, D. C. Lupascu, Mechanical property measurements of heterogeneous materials by selective nanoindentation: Application to limn 2 o 4 cathode, Materials Science and Engineering: A 593 (2014) 92-102.

[18] R. L. Smith, G. E. Sandly, An accurate method of determining the hardness of metals, with particular reference to those of a high degree of hardness, Proceedings of the Institution of Mechanical Engineers 102 (1) (1922) 623641

[19] J. Marteau, S. Bouvier, M. Bigerelle, Review on numerical modeling of instrumented indentation tests for elastoplastic material behavior identification, Archives of Computational Methods in Engineering 22 (4) (2015) $577-593$.

[20] E. Herbert, G. Pharr, W. Oliver, B. Lucas, J. Hay, On the measurement 
of stress-strain curves by spherical indentation, Thin Solid Films 398-399 (2001) 331-335.

[21] N. Chollacoop, M. Dao, S. Suresh, Depth-sensing instrumented indentation with dual sharp indenters, Acta Materialia 51 (13) (2003) 3713 - 3729.

[22] N. A. Branch, G. Subhash, N. K. Arakere, M. A. Klecka, Materialdependent representative plastic strain for the prediction of indentation hardness, Acta Materialia 58 (19) (2010) 6487 - 6494.

[23] X. Hernot, C. Moussa, O. Bartier, Study of the concept of representative strain and constraint factor introduced by vickers indentation, Mechanics of Materials 68 (2014) $1-14$.

[24] L. Rabemananjara, X. Hernot, G. Mauvoisin, A. Gavrus, J.-M. Collin, Formulation of a representative plastic strain and representative plastic strain rate by using a conical indentation on a rigid visco-plastic material, Materials \& Design 68 (2015) 207 - 214.

[25] N. Ogasawara, N. Chiba, X. Chen, Representative strain of indentation analysis, Journal of Materials Research 20 (2005) 2225-2234.

[26] B. Taljat, T. Zacharia, F. Kosel, New analytical procedure to determine stress-strain curve from spherical indentation data, International Journal of Solids and Structures 35 (33) (1998) 4411 - 4426.

[27] Y. P. Cao, J. Lu, A new method to extract the plastic properties of metal materials from an instrumented spherical indentation loading curve, Acta Materialia 52 (13) (2004) 4023 - 4032.

[28] H. Lee, J. H. Lee, G. M. Pharr, A numerical approach to spherical indentation techniques for material property evaluation, Journal of the Mechanics and Physics of Solids 53 (9) (2005) $2037-2069$.

[29] M. Zhao, N. Ogasawara, N. Chiba, X. Chen, A new approach to measure the elastic-plastic properties of bulk materials using spherical indentation, Acta Materialia 54 (1) (2006) $23-32$. 
[30] N. Ogasawara, N. Chiba, X. Chen, A simple framework of spherical indentation for measuring elastoplastic properties, Mechanics of Materials 41 (9) (2009) $1025-1033$.

[31] J. H. Lee, T. Kim, H. Lee, A study on robust indentation techniques to evaluate elastic-plastic properties of metals, International Journal of Solids and Structures 47 (5) (2010) $647-664$.

[32] A. Giannakopoulos, S. Suresh, Determination of elastoplastic properties by instrumented sharp indentation, Scripta materialia 40 (10) (1999) 11911198 .

[33] T. Nakamura, T. Wang, S. Sampath, Determinátion of properties of graded materials by inverse analysis and instrumented indentation, Acta Materialia 48 (17) (2000) 4293-4306.

[34] T. Venkatesh, K. Van Vliet, A. Giannakopoulos, S. Suresh, Determination of elasto-plastic properties by instrumented sharp indentation: guidelines for property extraction, Scripta materialia 42 (9) (2000) 833-839.

[35] T. Capehart, Y. Cheng, Determining constitutive models from conical indentation: Sensitivity analysis, Journal of materials research 18 (04) (2003) $827-832$.

[36] C. Moussa, X. Hernot, O. Bartier, G. Delattre, G. Mauvoisin, Evaluation of the tensile properties of a material through spherical indentation: definition of an average representative strain and a confidence domain, Journal of Materials Science 49 (2) (2014) 592-603.

[37] C. Moussa, O. Bartier, X. Hernot, G. Mauvoisin, J.-M. Collin, G. Delattre, Mechanical characterization of carbonitrided steel with spherical indentation using the average representative strain, Materials \& Design 89 (2016) $1191-1198$. 
[38] X. Chen, J. J. Vlassak, Numerical study on the measurement of thin film mechanical properties by means of nanoindentation, Journal of Materials Research 16 (10) (2001) 2974-2982.

[39] K. Tho, S. Swaddiwudhipong, Z. Liu, K. Zeng, J. Hua, Uniqueness of reverse analysis from conical indentation tests, Journal of Materials Research 19 (2004) 2498-2502.

[40] S. Swaddiwudhipong, K. Tho, Z. Liu, K. Zeng, Material characterization based on dual indenters, International Journal of Solids and Structures 42 (1) (2005) $69-83$.

[41] J. Alkorta, J. Martinez-Esnaola, J. G. Sevillano, Absence of one-to-one correspondence between elastoplastic properties and sharp-indentation loadpenetration data, Journal of materials research 20 (02) (2005) 432-437.

[42] X. Chen, N. Ogasawara, M. Zhao, N. Chiba, On the uniqueness of measuring elastoplastic properties from indentation: The indistinguishable mystical materials, Journal of the Mechanics and Physics of Solids 55 (8) (2007) $1618-1660$.

[43] O. Bartier, X. Hernot, G. Mauvoisin, Theoretical and experimental analysis of contact radius for spherical indentation, Mechanics of Materials 42 (6) (2010) $640-656$.

[44] C. Ullner, E. Reimann, H. Kohlhoff, A. Subaric-Leitis, Effect and measurement of the machine compliance in the macro range of instrumented indentation test, Measurement 43 (2) (2010) 216 - 222.

[45] S.-K. Kang, Y.-C. Kim, Y.-H. Lee, J.-Y. Kim, D. Kwon, Determining effective radius and frame compliance in spherical nanoindentation, Materials Science and Engineering: A 538 (0) (2012) 58 - 62.

[46] K. J. Van Vliet, L. Prchlik, J. F. Smith, Direct measurement of indentation frame compliance, Journal of Materials Research 19 (2004) 325-331. 
[47] W. Li, H. Bei, J. Qu, Y. Gao, Effects of machine stiffness on the loadingdisplacement curve during spherical nano-indentation, Journal of Materials Research 28 (2013) 1903-1911.

[48] M. Y. N'jock, D. Chicot, J. Ndjaka, J. Lesage, X. Decoopman, F. Roudet, A. Mejias, A criterion to identify sinking-in and piling-up in indentation of materials, International Journal of Mechanical Sciences 90 (2015) 145 150 .

[49] D. Chicot, M. Y. N'Jock, F. Roudet, X. Decoopman, M. Staia, E. PuchiCabrera, Some improvements for determining the hardness of homogeneous materials from the work-of-indentation, International Journal of Mechanical Sciences 105 (2016) $279-290$.

[50] M. Lichinchi, C. Lenardi, J. Haupt, R. Vitali, Simulation of Berkovich nanoindentation experiments on thin films using finite element method, Thin solid films 312 (1) (1998) 240-248.

[51] P. Grau, G. Berg, W. Fraenzel, H. Meinhard, Recording hardness testing. problems of measurement at small indentation depths, physica status solidi (a) 146 (1) (1994) 537-548.

[52] P. Brammer, O. Bartier, X. Hernot, G. Mauvoisin, S.-S. Sablin, An alternative to the determination of the effective zero point in instrumented indentation: use of the slope of the indentation curve at indentation load values, Materials \& Design 40 (2012) 356-363.

[53] P. Brammer, G. Mauvoisin, O. Bartier, X. Hernot, S.-S. Sablin, Influence of sample thickness and experimental device configuration on the spherical indentation of aisi 1095 steel, Journal of Materials Research 27 (2012) 7684.

[54] Y. Liu, B. Wang, M. Yoshino, S. Roy, H. Lu, R. Komanduri, Combined numerical simulation and nanoindentation for determining mechanical prop- 
erties of single crystal copper at mesoscale, Journal of the Mechanics and Physics of Solids 53 (12) (2005) 2718-2741.

[55] R. Mulford, R. J. Asaro, R. J. Sebring, Spherical indentation of ductile power law materials, Journal of Materials Research 19 (2004) 2641-2649.

[56] C. Moy, M. Bocciarelli, S. Ringer, G. Ranzi, Indentation and imprint mapping for the identification of material properties in multi-layered systems, Computational Materials Science 50 (5) (2011) 1681 - 1691.

[57] K. Matsuda, Prediction of stress-strain curves of elastic-plastic/materials based on the vickers indentation, Philosophical Magazine A 82 (10) (2002) 1941-1951.

[58] M. Bocciarelli, V. Buljak, C. Moy, S. Ringer, G. Ranzi, An inverse analysis approach based on a $\{\mathrm{POD}\}$ direct model for the mechanical characterization of metallic materials, Computational Materials Science 95 (0) (2014) $302-308$.

[59] M. Bocciarelli, G. Bolzon, Indentation and imprint mapping for the identification of constitutive parameters of thin layers on substrate: perfectly bonded interfaces, Materials Science and Engineering: A 448 (1) (2007) 303-314.

[60] M. Boeciarelli, G. Maier, Indentation and imprint mapping method for identification of residual stresses, Computational Materials Science 39 (2) (2007) 381-392.

[61] S. Russell, P. Norvig, A. Intelligence, A modern approach, Artificial Intelligence. Prentice-Hall, Egnlewood Cliffs 25 (1995) 27.

[62] B. Raghavan, P. Breitkopf, Y. Tourbier, P. Villon, Towards a space reduction approach for structural shape optimization, Structural \& Multidisciplinary Optimization 48 (5) (2013) 987-1000. doi:10.1007/s00158-0130942-5. 
[63] B. Raghavan, L. Xia, P. Breitkopf, A. Rassineux, P. Villon, Towards simultaneous reduction of both input and output spaces for interactive simulation-based structural design, Computer Methods in Applied Mechanics and Engineering 265 (2013) 174-185.

[64] L. Meng, P. Breitkopf, B. Raghavan, G. Mauvoisin, O. Bartier, X. Hernot, Identification of material properties using indentation test and shape manifold learning approach, Computer Methods in Applied Mechanics and Engineering 297 (2015) 239-257.

[65] D. González, E. Cueto, F. Chinesta, Computational patient avatars for surgery planning, Annals of biomedical engineering 44 (1) (2016) 35-45.

[66] L. Meng, P. Breitkopf, G. L. Quilliec, B. Raghavan, P. Villon, Nonlinear shape-manifold learning approach: Concepts, tools and applications, Archives of Computational Methods in Engineering (2016) 121.doi:10.1007/s11831-016-9189-9.

[67] L. Xia, B. Raghavan, P. Breitkopf, W. Zhang, Numerical material representation using proper orthogonal decomposition and diffuse approximation, Applied Mathematics and Computation 224 (2013) 450-462.

[68] G. Le Quilliec, B. Raghavan, P. Breitkopf, A manifold learning-based reduced order model for springback shape characterization and optimization in sheet metal forming, Computer Methods in Applied Mechanics and Engineering 285 (2015) 621-638.

[69] B. Raghavan, M. Hamdaoui, M. Xiao, P. Breitkopf, P. Villon, A bi-level meta-modeling approach for structural optimization using modified POD bases and diffuse approximation, Computers \& Structures 127 (In Press, 2012) 19-28.

[70] J.-M. Collin, G. Mauvoisin, O. Bartier, R. E. Abdi, P. Pilvin, Experimental evaluation of the stress-strain curve by continuous indentation using 
different indenter shapes, Materials Science and Engineering: A 501 (1-2) (2009) 140-145.

[71] K. E. Prasad, N. Chollacoop, U. Ramamurty, Role of indenter angle on the plastic deformation underneath a sharp indenter and on representative strains: An experimental and numerical study, Acta Materialia 59 (11) (2011) $4343-4355$.

[72] Z. Song, K. Komvopoulos, Elastic-plastic spherical indentation: Deformation regimes, evolution of plasticity, and hardening effect, Mechanics of Materials 61 (2013) $91-100$.

[73] A. Nayebi, O. Bartier, G. Mauvoisin, R. E. Abdí, New method to determine the mechanical properties of heat treated steels, International Journal of Mechanical Sciences 43 (11) (2001) 2679-2697.

[74] A. Nayebi, R. E. Abdi, O. Bartier, G. Mauvoisin, New procedure to determine steel mechanical parameters from the spherical indentation technique, Mechanics of Materials 34 (4) (2002) $243-254$.

[75] D. Hibbitt, B. Karlsson, P. Sorensen, Abaqus analysis user's manual, Pawtucket, USA.

[76] S. Bulychev, V. Alekhin, M. Shorshorov, A. Ternovskii, G. Shnyrev, Determining Young's modulus from the indentor penetration diagram, Zavod. Lab. 41 (1975) $137-140$.

[77] S. Bulychev, V. Alekhin, M. Shorshorov, A. Ternovskii, Mechanical properties of materials studied from kinetic diagrams of load versus depth of impression during microimpression, Strength of Materials 8 (9) (1976) 10841089.

[78] M. Shorshorov, S. Bulychev, V. Alekhin, Work of plastic and elastic deformation during indenter indentation, Doklady Physics 26 (1981) 769-771. 
[79] J. L. Hay, P. J. Wolff, Small correction required when applying the Hertzian contact model to instrumented indentation data, Journal of Materials Research 16 (2001) 1280-1286.

[80] W. Oliver, G. Pharr, An improved technique for determining hardness and elastic modulus using load and displacement sensing indentation experiments, Journal of Materials Research 7 (1992) 1564-1583.

[81] Y.-T. Cheng, C.-M. Cheng, Scaling, dimensional analysis, and indentation measurements, Materials Science and Engineering: R: Reports 44 (4) (2004) 91-149.

[82] L. Meng, P. Breitkopf, G. L. Quilliec, An insight into the identifiability of material properties by instrumented indentation test using manifold approach based on p-h curve and imprint shape, International Journal of Solids and Structures 106-107 (2017) 13 - 26. doi:10.1016/j.ijsolstr.2016.12.002.

[83] M. D. McKay, R. J. Beckman, W. J. Conover, A comparison of three methods for selecting values of input variables in the analysis of output from a computer code, Technometrics 42 (1) (2000) 55-61. 